\title{
SEMI-RIEMANNIAN SUBMERSIONS WITH TOTALLY GEODESIC FIBRES
}

\author{
GABRIEL BĂDIŢOIU
}

\begin{abstract}
We classify semi-Riemannian submersions with connected totally geodesic fibres from a real pseudo-hyperbolic space onto a semi-Riemannian manifold under the assumption that the dimension of the fibres is less than or equal to three and the metrics induced on fibres are negative definite. Also, we obtain the classification of semiRiemannian submersions with connected complex totally geodesic fibres from a complex pseudo-hyperbolic space onto a semi-Riemannian manifold under the assumption that the dimension of the fibres is less than or equal to two and the metric induced on fibres are negative definite. We prove that there are no semi-Riemannian submersions with connected quaternionic fibres from a quaternionic pseudo-hyperbolic space onto a Riemannian manifold.
\end{abstract}

\section{INTRODUCTION AND MAIN RESULTS}

Riemannian submersions, introduced by O'Neill [One1] and Gray [Gra], have been used by many authors to construct specific Riemannian metrics. A systematic exposition can be found in Besse's book Bes. In this paper, we obtain classification results for semiRiemannian submersions with totally geodesic fibres.

We first recall briefly some related work on the classification problem of semi-Riemannian submersions. Escobales [Esc1, Esc2] and Ranjan [Ran1] classified Riemannian submersions with connected totally geodesic fibres from an $n$-sphere $S^{n}$, and with connected complex totally geodesic fibres from a complex projective $n$-space $\mathbb{C} P^{n}$, respectively. Ucci UcC] showed that there are no Riemannian submersions with fibres $\mathbb{C} P^{3}$ from the complex projective space $\mathbb{C} P^{7}$ onto $S^{8}(4)$, and with fibres $\mathbb{H} P^{1}$ from the quaternionic projective space $\mathbb{H} P^{3}$ onto $S^{8}(4)$. In [Ran2], Ranjan obtained a classification theorem for Riemannian submersions with connected totally geodesic fibres from a compact simple Lie group. Gromoll and Grove obtained in [G-G1] that, up to equivalence, the only Riemannian submersions of spheres (with connected fibres) are the Hopf fibrations, except possibly for fibrations of the 15 -sphere by homotopy 7 -spheres. This classification was invoked in the proof of the Diameter Rigidity Theorem (see [G-G2]) and of the Radius Rigidity Theorem (see Wil]). Using an approach different from Gromoll and Grove G-G1, Wilking Wilk] proved that a Riemannian submersion $\pi: S^{m} \rightarrow B^{b}$ is metrically equivalent to the Hopf

2000 Mathematics Subject Classification. Primary 53C50.

Key words and phrases. Semi-Riemannian submersions, isotropic semi-Riemannian manifolds, totally geodesic submanifolds, Ehresmann connections. 
fibration for $(m, b)=(15,8)$ and obtained an improved version of the Diameter Rigidity Theorem as a consequence of his classification theorem.

In comparison, there are few classification results for semi-Riemannian submersions, and the consequences seem to be at least as important as those for Riemannian submersions. In Mag, Magid proved that the only semi-Riemannian submersions with totally geodesic fibres from an anti-de Sitter space onto a Riemannian manifold are the canonical semiRiemannian submersions $H_{1}^{2 m+1} \rightarrow \mathbb{C} H^{m}$. In [Ba-Ia], the present author and Stere Ianuş classified semi-Riemannian submersions with connected totally geodesic fibres from a pseudo-hyperbolic space onto a Riemannian manifold, and with connected complex totally geodesic fibres from a complex pseudo-hyperbolic space onto a Riemannian manifold.

The aim of this work is to prove new classification results in the theory of semiRiemannian submersions analogous to those in Riemannian geometry. It is my pleasure to thank Professor Stere Ianuş for useful discussions on this subject.

Now, we list the main results proved in this paper.

Theorem 1.1. Let $\pi: H_{s+r^{\prime}}^{n+r} \rightarrow B_{s}^{n}$ be a semi-Riemannian submersion with connected totally geodesic fibres from a pseudo-hyperbolic space onto a semi-Riemannian manifold. If the dimension of the fibres is less than or equal to 3 and if the metrics induced on fibres are negative definite, then $\pi$ is equivalent to one of the following canonical semi-Riemannian submersions:

(a) $H_{2 t+1}^{2 m+1} \rightarrow \mathbb{C} H_{t}^{m}, \quad 0 \leq t \leq m$.

(b) $H_{4 t+3}^{4 m+3} \rightarrow \mathbb{H} H_{t}^{m}, \quad 0 \leq t \leq m$.

Theorem 1.2. Let $\pi: H_{s+r^{\prime}}^{n+r} \rightarrow B_{s}^{n}$ be a semi-Riemannian submersion with connected totally geodesic fibres from a pseudo-hyperbolic space onto a semi-Riemannian manifold. Assume that one of the following conditions is satisfied:

(A) $B$ is an isotropic semi-Riemannian manifold, which means that for any $x \in B_{s}^{n}$ and any real number $t$, the group of isometries $\mathbf{I}\left(B_{s}^{n}, g^{\prime}\right)$ preserving $x$ acts transitively on the set of all nonzero tangent vectors $X$ at $x$ for which $g^{\prime}(X, X)=t$, or

(B) index $(B) \in\{0, \operatorname{dim} B\}$.

Then $\pi$ is equivalent to one of the following canonical semi-Riemannian submersions:

(a) $H_{2 t+1}^{2 m+1} \rightarrow \mathbb{C} H_{t}^{m}, \quad 0 \leq t \leq m$.

(b) $H_{4 t+3}^{4 m+3} \rightarrow \mathbb{H} H_{t}^{m}, \quad 0 \leq t \leq m$.

(c) $H_{7+8 t}^{15} \rightarrow H_{8 t}^{8}(-4), \quad t \in\{0,1\}$.

Theorem 1.3. Let $\pi: \mathbb{C H}_{s}^{n} \rightarrow B$ be a semi-Riemannian submersion from a complex pseudo-hyperbolic space onto a semi-Riemannian manifold. Assume that the fibres are connected complex totally geodesic submanifolds, and one of the following conditions is satisfied:

(A) The real dimension of the fibres is $r \leq 2$ and the fibres are negative definite, or 
(B) $B$ is an isotropic semi-Riemannian manifold, or

(C) index $(B) \in\{0, \operatorname{dim} B\}$.

Then $\pi$ is equivalent to the canonical semi-Riemannian submersion

$$
\mathbb{C} H_{2 t+1}^{2 m+1} \rightarrow \mathbb{H} H_{t}^{m}, \quad 0 \leq t \leq m .
$$

Theorem 1.4. There exist no semi-Riemannian submersions $\pi: \mathbb{H} H_{s}^{n} \rightarrow B$ with connected quaternionic fibres from a quaternionic pseudo-hyperbolic space onto an isotropic semi-Riemannian manifold or onto a semi-Riemannian manifold of $\operatorname{ind} \operatorname{dex}(B) \in\{0, \operatorname{dim}(B)\}$.

\section{Preliminaries AND Examples}

In this section we recall several notions and results which will be needed throughout the paper. We also exhibit the construction of canonical semi-Riemannian submersions.

Definition 2.1. Let $(M, g)$ be an $(n+r)$-dimensional connected semi-Riemannian manifold of index $s+r^{\prime}$, and $\left(B, g^{\prime}\right)$ an $n$-dimensional connected semi-Riemannian manifold of index $s$, where $0 \leq s \leq n, 0 \leq r^{\prime} \leq r$. A semi-Riemannian submersion (see [One2]) is a smooth map $\pi: M \rightarrow B$ which is surjective and satisfies the following axioms:

(a) $\left.\pi_{*}\right|_{p}$ is surjective for all $p \in M$;

(b) the fibres $\pi^{-1}(b), b \in B$, are semi-Riemannian submanifolds of $M$;

(c) $\pi_{*}$ preserves scalar products of vectors normal to fibres.

We shall always assume that the fibres are connected, the dimension of the fibres $\operatorname{dim} M-\operatorname{dim} B>0$ and $\operatorname{dim} B>0$. The vectors tangent to fibres are called vertical and those normal to fibres are called horizontal. We denote by $\mathcal{V}$ the vertical distribution and by $\mathcal{H}$ the horizontal distribution.

The geometry of semi-Riemannian submersions is characterized by O'Neill's tensors $T$, $A$ (see [One1, One2]) defined for vector fields $E, F$ on $M$ by

$$
\begin{aligned}
& A_{E} F=h \nabla_{h E} v F+v \nabla_{h E} h F, \\
& T_{E} F=h \nabla_{v E} v F+v \nabla_{v E} h F,
\end{aligned}
$$

where $\nabla$ is the Levi-Civita connection of $g$, and $v$ and $h$ denote the orthogonal projections on $\mathcal{V}$ and $\mathcal{H}$, respectively. For basic properties of O'Neill's tensors see [One1, [One2], Bes] or [Ian.

Definition 2.2. (i) A vector field $X$ on $M$ is said to be basic if $X$ is horizontal and $\pi$-related to a vector field $X^{\prime}$ on $B$.

(ii) A vector field $X$ along the fibre $\pi^{-1}(x), x \in B$, is said to be basic along $\pi^{-1}(x)$ if $X$ is horizontal and $\pi_{* p} X(p)=\pi_{* q} X(q)$ for every $p, q \in \pi^{-1}(x)$.

We notice that each vector field $X^{\prime}$ on $B$ has a unique horizontal lift $X$ to $M$ which is basic. For a vertical vector field $V$ and a basic vector field $X$ we have $h \nabla_{V} X=A_{X} V$ 
(see [One1]). We denote by $R, R^{\prime}$ and $\hat{R}$ the Riemann curvature tensors of $M, B$ and of the fibre $\pi^{-1}(x), x \in M$, respectively. We choose the convention for the curvature tensor $R(E, F)=\nabla_{E} \nabla_{F}-\nabla_{F} \nabla_{E}-\nabla_{[E, F]}$. The Riemann curvature tensor is defined by

$$
R(E, F, G, H)=g(R(G, H) F, E) .
$$

For O'Neill's equations of a semi-Riemannian submersion we refer to [One1] or [Bes].

Definition 2.3. Two semi-Riemannian submersions $\pi, \pi^{\prime}:(M, g) \rightarrow\left(B, g^{\prime}\right)$ are said to be equivalent if there exists an isometry $f$ of $M$ which induces an isometry $\tilde{f}$ of $B$ so that $\pi^{\prime} \circ f=\tilde{f} \circ \pi$. The pair $(f, \tilde{f})$ is called a bundle isometry.

We shall need the following theorem, which is the semi-Riemannian version of Theorem 2.2 in Esc1].

Theorem 2.4. Let $\pi_{1}, \pi_{2}: M \rightarrow B$ be semi-Riemannian submersions from a complete connected semi-Riemannian manifold $M$ onto a semi-Riemannian manifold $B$. Assume that the fibres of these submersions are connected and totally geodesic and the metric induced on fibres is negative definite. Let $f$ be an isometry of $M$ satisfying the following properties at a given point $p \in M$ :

(1) $f_{* p}: T_{p} M \rightarrow T_{f(p)} M$ maps $\mathcal{H}_{1 p}$ onto $\mathcal{H}_{2 f(p)}$, where $\mathcal{H}_{i}$ denote the horizontal distributions of $\pi_{i}$ for $i \in\{1,2\}$.

(2) $f_{*} A_{1 E} F=A_{2 f_{*}} f_{*} F$ for every $E, F \in T_{p} M$, where $A_{i}$ are the integrability tensors associated with $\pi_{i}$.

Then $f$ induces an isometry $\tilde{f}$ of $B$ so that the pair $(f, \tilde{f})$ is a bundle isometry between $\pi_{1}$ and $\pi_{2}$. In particular, $\pi_{1}$ and $\pi_{2}$ are equivalent.

Escobales's proof of Theorem 2.2 in [Esc1, also works in this semi-Riemannian case. He proves that for any $b \in B$ which can be joined with $\pi_{1}(p)$ by a geodesic we have:

(i) for every $x \in \pi_{1}^{-1}(b), f_{* x}: T_{x} M \rightarrow T_{f(x)} M$ maps $\mathcal{H}_{1 x}$ onto $\mathcal{H}_{2 f(x)}$, and

(ii) $f$ maps the fibre $\pi_{1}^{-1}(b)$ into the fibre $\pi_{2}^{-1}\left(\pi_{2}(f(x))\right)$ with $x \in \pi_{1}^{-1}(b)$.

We notice that for any $x \in \pi_{1}^{-1}(b)$ with $b \in B$, which can be joined with $\pi_{1}(p)$ by a geodesic, the conditions (1) and (2) are also satisfied for the point $x$. Since $M$ is connected, $B$ is also connected. Therefore, any point $b \in B$ can be joined with $\pi_{1}(p)$ by a broken geodesic. Repeating the argument above, for any corner point of this broken geodesic, we see that for any $b \in B, f$ maps the fibre $\pi_{1}^{-1}(b)$ into a fibre.

Definition 2.5. Let $\langle\cdot, \cdot\rangle$ be the symmetric bilinear form on $\mathbb{R}^{m+1}$ given by

$$
\langle x, y\rangle=-\sum_{i=0}^{s} x_{i} y_{i}+\sum_{i=s+1}^{m} x_{i} y_{i}
$$


for $x=\left(x_{0}, \cdots, x_{m}\right), y=\left(y_{0}, \cdots, y_{m}\right) \in \mathbb{R}^{m+1}$. For any $c<0$ and any positive integer $s$, let $H_{s}^{m}(c)=\left\{x \in \mathbb{R}^{m+1} \mid\langle x, x\rangle=1 / c\right\}$ be the semi-Riemannian submanifold of $\mathbb{R}_{s+1}^{m+1}=\left(\mathbb{R}^{m+1}, d s^{2}=-d x^{0} \otimes d x^{0}-\cdots-d x^{s} \otimes d x^{s}+d x^{s+1} \otimes d x^{s+1}+\cdots+d x^{m} \otimes d x^{m}\right)$. $H_{s}^{m}(c)$ is called the $m$-dimensional (real) pseudo-hyperbolic space of index $s$.

We notice that $H_{s}^{m}(c)$ has constant sectional curvature $c$, whose curvature tensor is given by $R(X, Y, X, Y)=c\left(g(X, X) g(Y, Y)-g(X, Y)^{2}\right)$. We shall denote simply $H_{s}^{m}=$ $H_{s}^{m}(-1)$. It should be remarked that $H_{s}^{m}$ can be written as a homogeneous space, namely $H_{s}^{m}=S O(s+1, m-s) / S O(s, m-s), H_{2 s+1}^{2 m+1}=S U(s+1, m-s) / S U(s, m-s)$, and $H_{4 s+3}^{4 m+3}=S p(s+1, m-s) / S p(s, m-s)($ see [Wol] $)$.

Definition 2.6. Let $(\cdot, \cdot)$ be the Hermitian form on $\mathbb{C}^{m+1}$ given by

$$
(z, w)=-\sum_{i=0}^{s} z_{i} \bar{w}_{i}+\sum_{i=s+1}^{m} z_{i} \bar{w}_{i}
$$

for $z=\left(z_{0}, \cdots, z_{m}\right), w=\left(w_{0}, \cdots, w_{m}\right) \in \mathbb{C}^{m+1}$. For $c<0$, let $M(c)$ be the real hypersurface of $\mathbb{C}^{m+1}$ given by $M(c)=\left\{z \in \mathbb{C}^{m+1} \mid(z, z)=4 / c\right\}$, which is endowed with the induced metric of

$$
\left(\mathbb{C}^{m+1}, d s^{2}=-d z^{0} \otimes d \bar{z}^{0}-\cdots-d z^{s} \otimes d \bar{z}^{s}+d z^{s+1} \otimes d \bar{z}^{s+1}+\cdots+d z^{m} \otimes d \bar{z}^{m}\right) .
$$

The natural action of $S^{1}=\left\{e^{i \theta} \mid \theta \in \mathbb{R}\right\}$ on $\mathbb{C}^{m+1}$ induces an action on $M(c)$. Let $\mathbb{C} H_{s}^{m}(c)=M(c) / S^{1}$ endowed with the unique indefinite Kähler metric of index $2 s$ such that the projection $M(c) \rightarrow M(c) / S^{1}$ becomes a semi-Riemannian submersion (see $\left[\right.$ Ba-Ro]). $\mathbb{C} H_{s}^{m}(c)$ is called the complex pseudo-hyperbolic space.

Notice that $\mathbb{C} H_{s}^{m}(c)$ has constant holomorphic sectional curvature $c$, whose curvature tensor is given by $R(X, Y, X, Y)=(c / 4)\left(g(X, X) g(Y, Y)-g(X, Y)^{2}+3 g\left(I_{0} X, Y\right)^{2}\right)$, where $I_{0}$ is the natural complex structure on $\mathbb{C} H_{s}^{m}(c)$. We shall denote simply $\mathbb{C} H_{s}^{m}=$ $\mathbb{C} H_{s}^{m}(-4)$. It is well-known that $\mathbb{C} H_{s}^{m}$ is a homogeneous space, namely $\mathbb{C} H_{s}^{m}=S U(s+$ $1, m-s) / S(U(1) U(s, m-s))$ and $\mathbb{C} H_{2 s+1}^{2 m+1}=S p(s+1, m-s) / U(1) S p(s, m-s)$ (see Wol ).

We shall denote by $\mathbb{H} H_{s}^{n}$ the quaternionic pseudo-hyperbolic space of real dimension $4 n$, and of quaternionic index $s$ with quaternionic sectional curvature -4 , and by $S^{n}$ and $S^{n}(4)$ the spheres with sectional curvature 1 and 4 , respectively.

By a standard construction (see Theorem 9.80 in [Bes]), one can obtain many examples of semi-Riemannian submersions with totally geodesic fibres of type $\pi: G / K \rightarrow G / H$, where $G$ is a Lie group and $K, H$ are closed Lie subgroups of $G$ with $K \subset H$. In this way the following canonical semi-Riemannian submersions, also called generalized Hopf fibrations, are obtained: 
Example 1. Let $G=S U(t+1, m-t), H=S(U(1) U(t, m-t)), K=S U(t, m-t)$. For every $0 \leq t \leq m$, we have the semi-Riemannian submersion

$H_{2 t+1}^{2 m+1}=S U(t+1, m-t) / S U(t, m-t) \rightarrow \mathbb{C} H_{t}^{m}=S U(t+1, m-t) / S(U(1) U(t, m-t))$.

Example 2. Let $G=S p(t+1, m-t), H=S p(1) S p(t, m-t), K=S p(t, m-t)$. For every $0 \leq t \leq m$, we get the semi-Riemannian submersion

$$
H_{4 t+3}^{4 m+3}=S p(t+1, m-t) / S p(t, m-t) \rightarrow \mathbb{H} H_{t}^{m}=S p(t+1, m-t) / S p(1) S p(t, m-t) .
$$

Example 3. a) Let $G=\operatorname{Spin}(1,8), H=\operatorname{Spin}(8), K=\operatorname{Spin}(7)$. Then we have the semi-Riemannian submersion (see [Ba-Ia])

$$
H_{7}^{15}=\operatorname{Spin}(1,8) / \operatorname{Spin}(7) \rightarrow H^{8}(-4)=\operatorname{Spin}(1,8) / \operatorname{Spin}(8)
$$

b) Let $G=\operatorname{Spin}(9), H=\operatorname{Spin}(8), K=\operatorname{Spin}(7)$. Then we have the semi-Riemannian submersion (see $[\mathrm{Bes}]$ )

$$
S^{15}=\operatorname{Spin}(9) / \operatorname{Spin}(7) \rightarrow S^{8}(4)=\operatorname{Spin}(9) / \operatorname{Spin}(8)
$$

Example 4. Let $G=S p(t+1, m-t), H=S p(1) S p(t, m-t), K=U(1) S p(t, m-t)$. For every $0 \leq t \leq m$, we obtain the semi-Riemannian submersion

$\mathbb{C} H_{2 t+1}^{2 m+1}=S p(t+1, m-t) / U(1) S p(t, m-t) \rightarrow \mathbb{H} H_{t}^{m}=S p(t+1, m-t) / S p(1) S p(t, m-t)$.

In order to prove Theorem 1.2, we need the following nonexistence proposition, which is the semi-Riemannian version of Proposition 5.1 in [Ran1].

Proposition 2.7. There exist no semi-Riemannian submersions $\pi: H_{7+8 t}^{23} \rightarrow \mathbb{C} a H_{t}^{2}$, $t \in\{0,1,2\}$, with totally geodesic fibres from the 23-dimensional pseudo-hyperbolic space of index $7+8 t$ onto the Cayley pseudo-hyperbolic plane of Cayley index $t$.

We notice that the case $t=2$ is Proposition 5.1 in Ran1. For the case $t=0$, see Ba-Ia]. Here we only recall some details of Ranjan's proof and suggest its modification to the semi-Riemannian case. Ranjan's argument in [Ran1], which leads to a contradiction to the assumption of the existence of such a submersion, is based on finding for every $X \in \mathcal{H}_{p}, g(X, X) \neq 0$, an irreducible $C l\left(\mathcal{V}_{p}\right)$-submodule $S$ of $\mathcal{H}_{p}$ passing through $X$. Here $C l\left(\mathcal{V}_{p}\right)$ denotes the Clifford algebra of $\left(\mathcal{V}_{p}, \tilde{g}_{p}\right)$, where $\tilde{g}(U, V)=-g(U, V)$ for every $U, V \in \mathcal{V}_{p} . \mathcal{H}_{p}$ becomes a $C l\left(\mathcal{V}_{p}\right)$-module by considering the extension of the map $\mathcal{U}: \mathcal{V}_{p} \rightarrow \operatorname{End}\left(\mathcal{H}_{p}\right)$ defined by $\mathcal{U}(V)(X)=A_{X} V$ to the Clifford algebra $C l\left(\mathcal{V}_{p}\right)$. Since $\tilde{g}_{p}$ is positive definite, we have $C l\left(\mathcal{V}_{p}\right) \simeq \mathbb{R}(8) \oplus \mathbb{R}(8)$. Hence, $\mathcal{H}_{p}$ splits into two 8-dimensional irreducible $C l\left(\mathcal{V}_{p}\right)$-modules. Since the induced metrics on fibres are negative definite, we obtain in a manner similar to Ranjan's proof that

(i) for $g(X, X)>0, \pi^{-1}\left(\mathbb{C} a H^{1}\right)$ is totally geodesic in $H_{7+8 t}^{23}$ and is isometric to $H_{7}^{15}$, where $\mathbb{C} a H^{1}$ denotes the Cayley hyperbolic line through $\pi_{*} X$, and 
(ii) for $g(X, X)<0, \pi^{-1}\left(\mathbb{C} a H_{1}^{1}\right)$ is totally geodesic in $H_{7+8 t}^{23}$ and is isometric to $H_{15}^{15}$, where $\mathbb{C} a H_{1}^{1}$ denotes the negative definite Cayley hyperbolic line through $\pi_{*} X$.

We choose $S$ to be the horizontal space of the restricted submersion $\tilde{\pi}: H_{7}^{15} \rightarrow \mathbb{C} a H^{1}$ if $g(X, X)>0$ or $\tilde{\pi}: H_{15}^{15} \rightarrow \mathbb{C} a H_{1}^{1}$ if $g(X, X)<0$.

\section{Proof of the main Results}

The next lemma gives useful properties of O'Neill's integrability tensor.

Lemma 3.1. Let $\pi: M \rightarrow B$ be a semi-Riemannian submersion with connected totally geodesic fibres from a semi-Riemannian manifold $M$ with constant curvature $c \neq 0$. Then the following assertions are true:

(a) If $X$ is a horizontal vector such that $g(X, X) \neq 0$, then the map $A_{X}: \mathcal{V} \rightarrow \mathcal{H}$ given by $A_{X}(V)=A_{X} V$ is injective and the map $A_{X}^{*}: \mathcal{H} \rightarrow \mathcal{V}$ given by $A_{X}^{*}(Y)=A_{X} Y$ is surjective.

(b) If $X, Y$ are the horizontal liftings along the fibre $\pi^{-1}(\pi(p)), p \in M$, of two vectors $X^{\prime}, Y^{\prime} \in T_{\pi(p)} B$ respectively, $g^{\prime}\left(X^{\prime}, X^{\prime}\right) \neq 0$ and $\left(A_{X} Y\right)(p)=0$, then $A_{X} Y=0$ along the fibre $\pi^{-1}(\pi(p))$.

Proof. (a) By O'Neill's equations, we get

$$
g\left(A_{X} V, A_{X} W\right)=c g(X, X) g(V, W)
$$

for a horizontal vector field $X$ and for vertical vector fields $V$ and $W$. Thus $A_{X}^{*} A_{X} V=$ $-c g(X, X) V$ for every vertical vector field $V$. Therefore $A_{X}: \mathcal{V} \rightarrow \mathcal{H}$ is injective and $A_{X}^{*}: \mathcal{H} \rightarrow \mathcal{V}$ is surjective.

(b) By O’Neill's equations, we have

$$
-3 g\left(A_{X} Y, A_{X} Z\right)=c[g(X, X) g(Y, Z)-g(X, Y) g(X, Z)]-R^{\prime}\left(\pi_{*} X, \pi_{*} Y, \pi_{*} X, \pi_{*} Z\right)
$$

for horizontal vector fields $X, Y$ and $Z$.

If $X, Y, Z$ are basic vector fields, then $g\left(A_{X} Y, A_{X} Z\right)$ is constant along the fibre $\pi^{-1}(\pi(p))$. Therefore, $g\left(A_{X} A_{X} Y, Z\right)=0$ along the fibre $\pi^{-1}(\pi(p))$ for every basic vector field $Z$. Hence $A_{X} A_{X} Y=0$ along $\pi^{-1}(\pi(p))$. Since $A_{X}: \mathcal{V} \rightarrow \mathcal{H}$ is injective, it follows that $A_{X} Y=0$ along the fibre $\pi^{-1}(\pi(p))$.

Lemma 3.2. If $\pi: M \rightarrow B$ is a semi-Riemannian submersion with connected totally geodesic fibres from a semi-Riemannian manifold $M$ with constant curvature $c \neq 0$ onto a semi-Riemannian manifold $B$, then the tangent bundle of any fibre is trivial.

Proof. Let $x \in B$ and $p \in \pi^{-1}(x)$. Let $\left\{v_{1 p}, \ldots, v_{r p}\right\}$ be an orthonormal basis in $\mathcal{V}_{p}$. Let $Y_{1}, Y_{2}, \ldots, Y_{r}$ be the horizontal liftings along the fibre $\pi^{-1}(\pi(p))$ of $(1 /(c g(X, X))) \pi_{*} A_{X} v_{1 p}$, 
$(1 /(c g(X, X))) \pi_{*} A_{X} v_{2 p}, \ldots,(1 /(c g(X, X))) \pi_{*} A_{X} v_{r p}$, respectively. Let $v_{i}=A_{X} Y_{i}$ for each $i \in\{1, \ldots, r\}$. Since

$$
\begin{aligned}
g\left(v_{j}, v_{l}\right) & =g\left(A_{X} Y_{j}, A_{X} Y_{l}\right) \\
& =(1 / 3)\left(R^{\prime}\left(\pi_{*} X, \pi_{*} Y_{j}, \pi_{*} X, \pi_{*} Y_{l}\right)-c g(X, X) g\left(Y_{j}, Y_{l}\right)+c g\left(X, Y_{j}\right) g\left(X, Y_{l}\right)\right)
\end{aligned}
$$

is constant along the fibre $\pi^{-1}(\pi(p))$ and

$$
g\left(A_{X} Y_{j}, A_{X} Y_{l}\right)(p)=\frac{1}{c^{2}} g\left(A_{X} A_{X} v_{j p}, A_{X} A_{X} v_{l p}\right)=g(X, X)^{2} g\left(v_{j p}, v_{l p}\right)=\varepsilon_{j} \delta_{j l}
$$

we see that $\left\{v_{1}, v_{2}, \ldots, v_{r}\right\}$ is a global orthonormal basis of the tangent bundle of the fibre $\pi^{-1}(x)$, which makes the tangent bundle trivial.

We suppose that the curvature of the total space is negative. The case of positive curvature can be reduced to the negative one by changing simultaneously the signs of the metrics on the base and on the total space. We establish relations between the dimensions and the indices of fibres and of base spaces, and see how the geometry of base spaces looks like.

Theorem 3.3. Let $\pi: M \rightarrow B$ be a semi-Riemannian submersion with connected totally geodesic fibres from an $(n+r)$-dimensional semi-Riemannian manifold $M$ of index $s+r^{\prime}$ with constant negative curvature $c$ onto an $n$-dimensional semi-Riemannian manifold $B$ of index s. Then the following hold:

(1) $n=k(r+1)$ for some positive integer $k$ and $s=q_{1}\left(r^{\prime}+1\right)+q_{2}\left(r-r^{\prime}\right)$ for some nonnegative integers $q_{1}, q_{2}$ with $q_{1}+q_{2}=k$.

(2) If, moreover, $M$ is a simply connected complete semi-Riemannian manifold and the dimension of fibres is less than or equal to 3 and the metric induced on fibres is negative definite, then $B$ is an isotropic semi-Riemannian manifold and $r \in\{1,3\}$.

Proof. Normalizing the metric on $M$, we can suppose $c=-1$. Let $p \in M$. Since the tangent bundle of the fibre $\pi^{-1}(\pi(p))$ is trivial, we can choose a global orthonormal frame $\left\{v_{1}, v_{2}, \ldots, v_{r}\right\}$ for the tangent bundle of $\pi^{-1}(\pi(p))$. We have $g\left(v_{i}, v_{j}\right)=\varepsilon_{i} \delta_{i j}, \varepsilon_{i} \in\{-1,1\}$, and $\operatorname{card}\left\{i \mid \varepsilon_{i}<0\right\}=r^{\prime}$.

(1) Let $X$ be the horizontal lifting along the fibre $\pi^{-1}(\pi(p))$ of a vector $X^{\prime} \in T_{\pi(p)} B$, so that $g\left(X^{\prime}, X^{\prime}\right) \in\{-1,1\}$. By O'Neill's equations, we have

$$
g\left(A_{Y} V, A_{Y} V\right)=-g(Y, Y) g(V, V)
$$

for a horizontal vector field $Y$ and for a vertical vector field $V$. Along the fibre $\pi^{-1}(\pi(p))$ we obtain for every $i, j \in\{1, \ldots, r\}$

$$
\begin{gathered}
g\left(A_{X} v_{i}, A_{X} v_{j}\right)=-g(X, X) g\left(v_{i}, v_{j}\right)=-g(X, X) \varepsilon_{i} \delta_{i j} \\
g\left(X, A_{X} v_{i}\right)=-g\left(A_{X} X, v_{i}\right)=0 .
\end{gathered}
$$

Thus $\left\{X, A_{X} v_{1}, \ldots, A_{X} v_{r}\right\}$ is an orthonormal system. Hence $n \geq r+1$. 
Let $L_{0}=X$. For every integer $\alpha$ such that $1 \leq \alpha<n /(r+1)$, let $L_{\alpha}$ be a horizontal vector field along the fibre $\pi^{-1}(\pi(p))$ such that $L_{\alpha}$ is the horizontal lifting of some unit vector (i.e., $\left.g\left(L_{\alpha}, L_{\alpha}\right) \in\{-1,1\}\right)$, that $L_{\alpha}$ is orthogonal to $L_{0}, L_{1}, \ldots, L_{\alpha-1}$ and that $L_{\alpha}(p) \in \operatorname{ker} A_{L_{0}(p)}^{*} \cap \operatorname{ker} A_{L_{1}(p)}^{*} \cap \cdots \cap \operatorname{ker} A_{L_{\alpha-1}(p)}^{*}$. Then, by Lemma 3.1, $L_{\alpha}(q)$ belongs to ker $A_{L_{0}(q)}^{*} \cap \operatorname{ker} A_{L_{1}(q)}^{*} \cap \cdots \cap \operatorname{ker} A_{L_{\alpha-1}(q)}^{*}$ for every $q \in \pi^{-1}(\pi(p))$. Therefore, for $j \in$ $\{1, \ldots, r\}$ and $\alpha, \beta \geq 0$, we get

$$
g\left(A_{L_{\alpha}} v_{j}, L_{\beta}\right)=-g\left(v_{j}, A_{L_{\alpha}} L_{\beta}\right)=0
$$

along the fibre $\pi^{-1}(\pi(p))$.

By O'Neill's equations, we obtain

$$
\begin{aligned}
R(X, U, Y, V) & =g\left(\left(\nabla_{U} A\right)_{X} Y, V\right)+g\left(A_{X} U, A_{Y} V\right) \\
& =g\left(\nabla_{U} A_{X} Y, V\right)-g\left(A_{\nabla_{U}} Y, V\right)-g\left(A_{X} \nabla_{U} Y, V\right)+g\left(A_{X} U, A_{Y} V\right) \\
& =g\left(\nabla_{U} A_{X} Y, V\right)+g\left(A_{Y} A_{X} U, V\right)-g\left(A_{X} A_{Y} U, V\right)-g\left(A_{Y} A_{X} U, V\right) \\
& =g\left(\nabla_{U} A_{X} Y, V\right)+g\left(A_{Y} U, A_{X} V\right)
\end{aligned}
$$

for basic vector fields $X, Y$ and for vertical vector fields $U, V$. Thus, along the fibre $\pi^{-1}(\pi(p))$ we get for every $\alpha, \beta \geq 0$ and $j, l \in\{1, \ldots, r\}$

$$
\begin{aligned}
g\left(A_{L_{\alpha}} v_{j}, A_{L_{\beta}} v_{l}\right) & =R\left(L_{\alpha}, v_{l}, L_{\beta}, v_{j}\right)-g\left(\nabla_{v_{l}} A_{L_{\alpha}} L_{\beta}, v_{j}\right) \\
& =-g\left(L_{\alpha}, L_{\beta}\right) g\left(v_{l}, v_{j}\right)-v_{l}\left(g\left(A_{L_{\alpha}} L_{\beta}, v_{j}\right)\right)+g\left(A_{L_{\alpha}} L_{\beta}, \nabla_{v_{l}} v_{j}\right) .
\end{aligned}
$$

Since $A_{L_{\alpha}} L_{\beta}=0$ along the fibre $\pi^{-1}(\pi(p))$, it follows that

$$
g\left(A_{L_{\alpha}} v_{j}, A_{L_{\beta}} v_{l}\right)=-g\left(L_{\alpha}, L_{\beta}\right) g\left(v_{l}, v_{j}\right)=-g\left(L_{\alpha}, L_{\beta}\right) \varepsilon_{l} \delta_{l j} .
$$

We proved that for some positive integer $k$,

$$
\mathcal{L}=\left\{L_{0}, A_{L_{0}} v_{1}, \ldots, A_{L_{0}} v_{r}, \ldots, L_{k-1}, A_{L_{k-1}} v_{1}, \ldots, A_{L_{k-1}} v_{r}\right\}
$$

is an orthonormal basis of $\mathcal{H}$ along the fibre $\pi^{-1}(\pi(p))$. Thus $\operatorname{dim} B=(1+\operatorname{dim}$ fibre $) k$ for some positive integer $k$. Counting the timelike vectors in $\mathcal{L}$, we get index $(B)=$ $q_{1}\left(r^{\prime}+1\right)+q_{2}\left(r-r^{\prime}\right)$ for some nonnegative integers $q_{1}, q_{2}$ with $q_{1}+q_{2}=k$.

(2) Let $x \in B$ and $X^{\prime}, Y^{\prime} \in T_{x} B$ such that $g^{\prime}\left(X^{\prime}, X^{\prime}\right)=g^{\prime}\left(Y^{\prime}, Y^{\prime}\right) \neq 0$. We shall construct an isometry $\tilde{f}: B \rightarrow B$ such that $\tilde{f}(x)=x$ and $\tilde{f}_{*} X^{\prime}=Y^{\prime}$. Note that we may assume that $g^{\prime}\left(X^{\prime}, X^{\prime}\right)=g^{\prime}\left(Y^{\prime}, Y^{\prime}\right)= \pm 1$. Let $X, Y$ be the horizontal liftings along the fibre $\pi^{-1}(x)$ of $X^{\prime}$ and $Y^{\prime}$, respectively. Take $p \in \pi^{-1}(x)$. Let

$$
\begin{aligned}
& \mathcal{L}=\left\{L_{0}, A_{L_{0}} v_{1}, \ldots, A_{L_{0}} v_{r}, \ldots, L_{k-1}, A_{L_{k-1}} v_{1}, \ldots, A_{L_{k-1}} v_{r}\right\}, \\
& \mathcal{L}^{\prime}=\left\{L_{0}^{\prime}, A_{L_{0}^{\prime}} v_{1}^{\prime}, \ldots, A_{L_{0}^{\prime}} v_{r}^{\prime}, \ldots, L_{k-1}^{\prime}, A_{L_{k-1}^{\prime}} v_{1}^{\prime}, \ldots, A_{L_{k-1}^{\prime}} v_{r}^{\prime}\right\}
\end{aligned}
$$

be two orthonormal bases constructed as above such that $L_{0}=X, L_{0}^{\prime}=Y, g\left(L_{\alpha}, L_{\alpha}\right)=$ $g\left(L_{\alpha}^{\prime}, L_{\alpha}^{\prime}\right)$ for $\alpha \in\{1, \ldots, k-1\}$, and that $\left\{v_{1}=A_{X} Y_{1}, \ldots, v_{r}=A_{X} Y_{r}\right\}$ and $\left\{v_{1}^{\prime}=\right.$ $\left.A_{Y} Y_{1}^{\prime}, \ldots, v_{r}^{\prime}=A_{Y} Y_{r}^{\prime}\right\}$ are orthonormal bases of the tangent bundle of the fibre $\pi^{-1}(\pi(p))$, 
where $Y_{1}, \ldots, Y_{r}$ and $Y_{1}^{\prime}, \ldots, Y_{r}^{\prime}$ are the horizontal liftings along $\pi^{-1}(\pi(p))$ of the vectors $\pi_{*} A_{X} v_{1 p}, \ldots, \pi_{*} A_{X} v_{r p}$ and $\pi_{*} A_{Y} v_{1 p}^{\prime}, \ldots, \pi_{*} A_{Y} v_{r p}^{\prime}$, respectively (as in Lemma 3.1), for which $g\left(v_{i}, v_{j}\right)=g\left(v_{i}^{\prime}, v_{j}^{\prime}\right)$ for $i, j \in\{1, \cdots, r\}$. Let $\phi: T_{p} M \rightarrow T_{p} M$ be the linear map given by $\phi\left(L_{\alpha}\right)=L_{\alpha}^{\prime}, \phi\left(v_{j}\right)=v_{j}^{\prime}, \phi\left(A_{L_{\alpha}} v_{j}\right)=A_{L_{\alpha}^{\prime}} v_{j}^{\prime}$ for every $\alpha \in\{0, \ldots, k-1\}$ and $j \in\{1, \ldots, r\}$. Since both $\mathcal{L}, \mathcal{L}^{\prime}$ are orthonormal bases, we see that $\phi$ is a linear isometry.

We shall apply Theorem 2.4. Thus we need to prove that $\phi\left(A_{E} F\right)=A_{\phi(E)} \phi(F)$ for every $E, F \in T_{p} M$. Indeed, we obtain for $\alpha, \beta \in\{0, \ldots, k-1\}$ and $j, l \in\{1, \ldots, r\}$,

$$
\begin{aligned}
\phi\left(A_{L_{\alpha}} L_{\beta}\right) & =\phi(0)=0=A_{L_{\alpha}^{\prime}} L_{\beta}^{\prime}=A_{\phi\left(L_{\alpha}\right)} \phi\left(L_{\beta}\right), \\
g\left(v_{j}, A_{L_{\alpha}} A_{L_{\beta}} v_{l}\right) & =-g\left(A_{L_{\alpha}} v_{j}, A_{L_{\beta}} v_{l}\right)=-g\left(L_{\alpha}, L_{\beta}\right) g\left(v_{j}, v_{l}\right) \\
& =-g\left(L_{\alpha}^{\prime}, L_{\beta}^{\prime}\right) g\left(v_{j}^{\prime}, v_{l}^{\prime}\right)=g\left(v_{j}^{\prime}, A_{L_{\alpha}^{\prime}} A_{L_{\beta}^{\prime}} v_{l}^{\prime}\right) .
\end{aligned}
$$

Hence $\phi\left(A_{L_{\alpha}} A_{L_{\beta}} v_{l}\right)=A_{\phi\left(L_{\alpha}\right)} \phi\left(A_{L_{\beta}} v_{l}\right)$.

Lemma 3.4. $A_{L_{\alpha}} v_{j}$ is a basic vector field along the fibre $\pi^{-1}(\pi(p))$ for every $1 \leq j \leq r$ and $\alpha \geq 0$.

Proof of Lemma 3.4. We have $g\left(A_{X} v_{j}, Z\right)=g\left(A_{X} A_{X} Y_{j}, Z\right)=-g\left(A_{X} Y_{j}, A_{X} Z\right)$. For every basic vector field $Z$ along the fibre $\pi^{-1}(\pi(p))$ we know that $g\left(A_{X} Y_{j}, A_{X} Z\right)$ is constant along the fibre $\pi^{-1}(\pi(p))$. Hence $A_{X} v_{j}$ is a basic vector field along the fibre $\pi^{-1}(\pi(p))$.

Now we assume $\alpha \geq 1$. Since $\operatorname{dim}\left(\operatorname{ker} A_{X}^{*} \cup \operatorname{ker} A_{L_{\alpha}}^{*}\right)=\operatorname{dim} \operatorname{ker} A_{X}^{*}+\operatorname{dim} \operatorname{ker} A_{L_{\alpha}}^{*}-$ $\operatorname{dim}\left(\operatorname{ker} A_{X}^{*} \cap \operatorname{ker} A_{L_{\alpha}}^{*}\right)=(n-r)+(n-r)-(n-2 r)=n$, it follows that ker $A_{X}^{*}+\operatorname{ker} A_{L_{\alpha}}^{*}=\mathcal{H}$. Hence $A_{L_{\alpha}} v_{j}$ is a basic vector field along the fibre $\pi^{-1}(\pi(p))$ if and only if the following conditions are satisfied: $g\left(A_{L_{\alpha}} v_{j}, Z_{1}\right)$ is constant along $\pi^{-1}(\pi(p))$ for every $Z_{1} \in \operatorname{ker} A_{X}^{*}$, which is a basic vector field along $\pi^{-1}(\pi(p))$, and $g\left(A_{L_{\alpha}} v_{j}, Z_{2}\right)$ is constant along the fibre $\pi^{-1}(\pi(p))$ for every $Z_{2} \in \operatorname{ker} A_{L_{\alpha}}^{*}$, which is a basic vector field along $\pi^{-1}(\pi(p))$. If $Z_{2} \in \operatorname{ker} A_{L_{\alpha}}^{*}$, then $A_{L_{\alpha}}^{*} Z_{2}=0$ along $\pi^{-1}(\pi(p))$. So $g\left(A_{L_{\alpha}} v_{j}, Z_{2}\right)=-g\left(v_{j}, A_{L_{\alpha}} Z_{2}\right)=0$ along $\pi^{-1}(\pi(p))$. If $Z_{1} \in \operatorname{ker} A_{X}^{*}$, then $A_{X}^{*} Z_{1}=0$ along $\pi^{-1}(\pi(p))$. By O'Neill's equations, we get along the fibre $\pi^{-1}(\pi(p))$

$$
\begin{aligned}
R^{\prime}\left(\pi_{*} X, \pi_{*} Y_{j}, \pi_{*} L_{\alpha}, \pi_{*} Z_{1}\right)= & R\left(X, Y_{j}, L_{\alpha}, Z_{1}\right)+2 g\left(A_{X} Y_{j}, A_{L_{\alpha}} Z_{1}\right) \\
& -g\left(A_{Y_{j}} L_{\alpha}, A_{X} Z_{1}\right)-g\left(A_{L_{\alpha}} X, A_{Y_{j}} Z_{1}\right) \\
= & -g\left(X, L_{\alpha}\right) g\left(Y_{j}, Z_{1}\right)+g\left(X, Z_{1}\right) g\left(Y_{j}, L_{\alpha}\right) \\
& +2 g\left(v_{j}, A_{L_{\alpha}} Z_{1}\right),
\end{aligned}
$$

since $A_{L_{\alpha}} X=-A_{X} L_{\alpha}=0$ and $A_{X} Z_{1}=0$. Hence $g\left(v_{j}, A_{L_{\alpha}} Z_{1}\right)=-g\left(A_{L_{\alpha}} v_{j}, Z_{1}\right)$ is constant along $\pi^{-1}(\pi(p))$ for every $Z_{1} \in \operatorname{ker} A_{X}^{*}$, which is a basic vector field along $\pi^{-1}(\pi(p))$.

We proved that $A_{L_{\alpha}} v_{j}$ is a basic vector field along $\pi^{-1}(\pi(p))$ for every $\alpha \geq 0$ and $j \in\{1, \ldots, r\}$.

We denote by $\hat{\nabla}$ the induced Levi-Civita connection on the fibre $\pi^{-1}(\pi(p))$.

Lemma 3.5. $A_{A_{L_{\alpha}} v_{i}} A_{L_{\beta}} v_{j}=g\left(L_{\alpha}, L_{\beta}\right) \hat{\nabla}_{v_{i}} v_{j}$. 
Proof of Lemma 3.5. By the relation (3.1) together with Lemma 3.4, we obtain for $i, j, l \in$ $\{1, \ldots, r\}$ and $\alpha, \beta \geq 0$ that

$$
\begin{aligned}
g\left(A_{A_{L_{\alpha}} v_{i}} A_{L_{\beta}} v_{j}, v_{l}\right)= & -g\left(A_{A_{L_{\alpha}} v_{i}} v_{l}, A_{L_{\beta}} v_{j}\right) \\
= & -R\left(L_{\beta}, v_{l}, A_{L_{\alpha}} v_{i}, v_{j}\right)+g\left(\nabla_{v_{l}} A_{L_{\beta}} A_{L_{\alpha}} v_{i}, v_{j}\right) \\
= & g\left(L_{\beta}, A_{L_{\alpha}} v_{i}\right) g\left(v_{l}, v_{j}\right)+v_{l} g\left(A_{L_{\beta}} A_{L_{\alpha}} v_{i}, v_{j}\right) \\
& -g\left(A_{L_{\beta}} A_{L_{\alpha}} v_{i}, \nabla_{v_{l}} v_{j}\right) \\
= & -v_{l} g\left(A_{L_{\alpha}} v_{i}, A_{L_{\beta}} v_{j}\right)+g\left(A_{L_{\alpha}} v_{i}, A_{L_{\beta}} v_{t}\right) g\left(\nabla_{v_{l}} v_{j}, v_{t}\right) \varepsilon_{t} \\
= & -g\left(L_{\alpha}, L_{\beta}\right) g\left(\hat{\nabla}_{v_{l}} v_{j}, v_{i}\right) \\
= & g\left(L_{\alpha}, L_{\beta}\right) g\left(\hat{\nabla}_{v_{i}} v_{j}, v_{l}\right) .
\end{aligned}
$$

In the last equality we used the fact that $v_{j}=A_{X} Y_{j}$ is a Killing vector field along the fibre $\pi^{-1}(\pi(p))$ (see [Bis] or [Bes]). Thus

$$
A_{A_{L_{\alpha}} v_{i}} A_{L_{\beta}} v_{j}=g\left(L_{\alpha}, L_{\beta}\right) \hat{\nabla}_{v_{i}} v_{j}
$$

Lemma 3.6. The following assertions are true:

(a) $r \neq 2$.

(b) If $r=1$, then $A_{A_{L_{\alpha}} v_{1}} A_{L_{\beta}} v_{1}=0$.

(c) If $r=3$ and if we set $v_{3 p}=\left(\hat{\nabla}_{v_{1}} v_{2}\right)(p)$, then $v_{3}=\hat{\nabla}_{v_{1}} v_{2}$ and

$$
g\left(\hat{\nabla}_{v_{i}} v_{j}, v_{k}\right)=\left\{\begin{array}{cl}
0 & \text { if two of } i, j, k \text { are equal, } \\
\varepsilon\left(\begin{array}{lll}
1 & 2 & 3 \\
i & j & k
\end{array}\right) g\left(v_{3}, v_{3}\right) & \text { if }\{i, j, k\}=\{1,2,3\},
\end{array}\right.
$$

where $\varepsilon\left(\begin{array}{lll}1 & 2 & 3 \\ i & j & k\end{array}\right)$ is the signature of the permutation $\left(\begin{array}{lll}1 & 2 & 3 \\ i & j & k\end{array}\right)$.

Proof of Lemma 3.6. Since $v_{1}, \ldots, v_{r}$ are Killing vector fields along $\pi^{-1}(\pi(p))$ and $g\left(v_{i}, v_{i}\right) \in$ $\{-1,1\}$ for every $i$, we get

$$
g\left(\hat{\nabla}_{v_{i}} v_{j}, v_{i}\right)=g\left(\hat{\nabla}_{v_{i}} v_{i}, v_{j}\right)=g\left(\hat{\nabla}_{v_{j}} v_{i}, v_{i}\right)=0
$$

for every $i, j \in\{1, \ldots, r\}$.

(a) The case $r=2$ is not possible. Indeed, if $r=2$, then the relation $g\left(\nabla_{v_{1}} v_{2}, v_{1}\right)=$ $g\left(\nabla_{v_{1}} v_{2}, v_{2}\right)=0$ implies $\nabla_{v_{1}} v_{2}=0$. On the other hand,

$$
g\left(\nabla_{v_{1}} v_{2}, \nabla_{v_{1}} v_{2}\right)=-g\left(\hat{\nabla}_{v_{1}} \hat{\nabla}_{v_{2}} v_{2}, v_{1}\right)+\hat{R}\left(v_{1}, v_{2}, v_{1}, v_{2}\right)=-g\left(v_{1}, v_{1}\right) g\left(v_{2}, v_{2}\right) \in\{-1,1\},
$$

since $\hat{\nabla}_{v_{2}} v_{2}=g(X, X)^{-1} A_{A_{X} v_{2}} A_{X} v_{2}=0$ and each fibre has constant curvature -1 . So we get a contradiction.

(b) If $r=1$, then $A_{A_{L_{\alpha}} v_{1}} A_{L_{\beta}} v_{1}=0$ for every $\alpha$ and $\beta$, because $0=A_{A_{X} v_{1}} A_{X} v_{1}=$ $g(X, X) \nabla_{v_{1}} v_{1}$ implies $\nabla_{v_{1}} v_{1}=0$. 
(c) In the case $r=3$ we shall prove $g\left(\hat{\nabla}_{v_{1}} v_{2}, v_{3}\right)$ is constant along the fibre $\pi^{-1}(\pi(p))$. Since O'Neill's integrability tensor $A$ is skew-symmetric, it follows that $\hat{\nabla}_{v_{i}} v_{j}=-\hat{\nabla}_{v_{j}} v_{i}$. Then $\hat{\nabla}_{v_{i}} v_{j}=(1 / 2)\left[v_{i}, v_{j}\right]$ is a Killing vector field along $\pi^{-1}(\pi(p))$. We then obtain

$$
\begin{aligned}
v_{1} g\left(\hat{\nabla}_{v_{1}} v_{2}, v_{3}\right) & =g\left(\hat{\nabla}_{v_{1}} \hat{\nabla}_{v_{1}} v_{2}, v_{3}\right)+g\left(\hat{\nabla}_{v_{1}} v_{2}, \hat{\nabla}_{v_{1}} v_{3}\right) \\
& =-g\left(\hat{\nabla}_{v_{3}} \hat{\nabla}_{v_{1}} v_{2}, v_{1}\right)+g\left(\hat{\nabla}_{v_{1}} v_{2}, \hat{\nabla}_{v_{1}} v_{3}\right) \\
& =-v_{3} g\left(\hat{\nabla}_{v_{1}} v_{2}, v_{1}\right)+g\left(\hat{\nabla}_{v_{1}} v_{2}, \hat{\nabla}_{v_{1}} v_{3}+\hat{\nabla}_{v_{3}} v_{1}\right)=0 .
\end{aligned}
$$

Analogously, we get $v_{2} g\left(\hat{\nabla}_{v_{1}} v_{2}, v_{3}\right)=-v_{2} g\left(\hat{\nabla}_{v_{2}} v_{1}, v_{3}\right)=0$. We also obtain

$$
v_{3} g\left(\hat{\nabla}_{v_{1}} v_{2}, v_{3}\right)=g\left(\hat{\nabla}_{v_{3}} \hat{\nabla}_{v_{1}} v_{2}, v_{3}\right)+g\left(\hat{\nabla}_{v_{1}} v_{2}, \hat{\nabla}_{v_{3}} v_{3}\right)=0
$$

since $\hat{\nabla}_{v_{3}} v_{3}=0$ and $\hat{\nabla}_{v_{1}} v_{2}$ is a Killing vector field along $\pi^{-1}(\pi(p))$. It is easy to see that

$$
\begin{aligned}
g\left(\hat{\nabla}_{v_{1}} v_{2}, v_{3}\right) & =-g\left(\hat{\nabla}_{v_{2}} v_{1}, v_{3}\right)=g\left(\hat{\nabla}_{v_{2}} v_{3}, v_{1}\right) \\
& =-g\left(\hat{\nabla}_{v_{3}} v_{2}, v_{1}\right)=g\left(\hat{\nabla}_{v_{3}} v_{1}, v_{2}\right)=-g\left(\hat{\nabla}_{v_{1}} v_{3}, v_{2}\right) .
\end{aligned}
$$

Thus $g\left(\hat{\nabla}_{v_{i}} v_{j}, v_{l}\right)$ is constant along the fibre $\pi^{-1}(\pi(p))$ for each $i, j, l \in\{1,2,3\}$. Therefore

$$
g\left(A_{X} A_{A_{X} v_{i}} A_{X} v_{j}, A_{X} v_{l}\right)=-g(X, X) g\left(A_{A_{X} v_{i}} A_{X} v_{j}, v_{l}\right)=-g(X, X)^{2} g\left(\hat{\nabla}_{v_{i}} v_{j}, v_{l}\right)
$$

is constant along $\pi^{-1}(\pi(p))$. Also, we compute for $\alpha \geq 1$

$$
\begin{gathered}
g\left(A_{X} A_{A_{X} v_{i}} A_{X} v_{j}, A_{L_{\alpha}} v_{l}\right)=-g\left(A_{A_{X} v_{i}} A_{X} v_{j}, A_{X} A_{L_{\alpha}} v_{l}\right)=0, \\
g\left(A_{X} A_{A_{X} v_{i}} A_{X} v_{j}, L_{\alpha}\right)=-g\left(A_{A_{X} v_{i}} A_{X} v_{j}, A_{X} L_{\alpha}\right)=0 .
\end{gathered}
$$

Hence $A_{X} A_{A_{X} v_{i}} A_{X} v_{j}=g(X, X) A_{X} \hat{\nabla}_{v_{i}} v_{j}$ is a basic vector field for each $i, j \in\{1, \ldots, r\}$.

We choose $v_{3 p}=\left(\hat{\nabla}_{v_{1}} v_{2}\right)(p)$. Since $A_{X} \hat{\nabla}_{v_{1}} v_{2}$ is a basic vector field along $\pi^{-1}(\pi(p))$, we get the horizontal lifting along $\pi^{-1}(\pi(p))$ of $\pi_{*}\left(A_{X} \hat{\nabla}_{v_{1}} v_{2}(p)\right)=\pi_{*} A_{X} v_{3 p}$ is $g(X, X)^{-1} A_{X} \hat{\nabla}_{v_{1}} v_{2}$. On the other hand, $Y_{3}$ is, by definition, the horizontal lifting of $g(X, X)^{-1} \pi_{*} A_{X} v_{3 p}$ along $\pi^{-1}(\pi(p))$. It follows that $Y_{3}=g(X, X)^{-1} A_{X} \hat{\nabla}_{v_{1}} v_{2}$ along $\pi^{-1}(\pi(p))$. Thus

$$
v_{3}=A_{X} Y_{3}=g(X, X)^{-1} A_{X} A_{X} \hat{\nabla}_{v_{1}} v_{2}=\hat{\nabla}_{v_{1}} v_{2}
$$

along the fibre $\pi^{-1}(\pi(p))$.

For $r=3$, we choose $v_{3 p}^{\prime}=\left(\hat{\nabla}_{v_{1}^{\prime}} v_{2}^{\prime}\right)(p)$. If we repeat the argument above for the basis $\left\{v_{1}^{\prime}, v_{2}^{\prime}, v_{3}^{\prime}\right\}$, by Lemma 3.6, we get $v_{3}^{\prime}=\hat{\nabla}_{v_{1}^{\prime}} v_{2}^{\prime}$ along the fibre $\pi^{-1}(\pi(p))$. It follows that $g\left(\hat{\nabla}_{v_{i}} v_{j}, v_{l}\right)=g\left(\hat{\nabla}_{v_{i}^{\prime}} v_{j}^{\prime}, v_{l}^{\prime}\right)$ for each $i, j, l \in\{1,2,3\}$.

Returning to the computation of $g\left(A_{A_{L_{\alpha}} v_{i}} A_{L_{\beta}} v_{j}, v_{l}\right)$, in both cases $r=1$ and $r=3$, we get for every $\alpha, \beta \geq 0$ and $i, j, k \in\{1, \ldots, r\}$

$$
\begin{aligned}
g\left(A_{A_{L_{\alpha}} v_{i}} A_{L_{\beta}} v_{j}, v_{l}\right) & =g\left(L_{\alpha}, L_{\beta}\right) g\left(\hat{\nabla}_{v_{i}} v_{j}, v_{l}\right) \\
& =g\left(L_{\alpha}^{\prime}, L_{\beta}^{\prime}\right) g\left(\hat{\nabla}_{v_{i}^{\prime}} v_{j}^{\prime}, v_{l}^{\prime}\right)=g\left(A_{A_{L_{\alpha}^{\prime}} v_{i}^{\prime}} A_{L_{\beta}^{\prime}} v_{j}^{\prime}, v_{l}^{\prime}\right) .
\end{aligned}
$$

Hence $\phi\left(A_{A_{L_{\alpha}} v_{i}} A_{L_{\beta}} v_{j}\right)=A_{\phi\left(A_{L_{\alpha}} v_{i}\right)} \phi\left(A_{L_{\beta}} v_{j}\right)$ and $\phi\left(A_{A_{L_{\alpha}} v_{i}} v_{j}\right)=A_{\phi\left(A_{L_{\alpha}} v_{i}\right)} \phi\left(v_{j}\right)$. 
By Corollary 2.3.14 in [Wol] we see that $\phi: T_{p} M \rightarrow T_{p} M$ extends to an isometry on $M$, denoted by $f: M \rightarrow M$, such that $f(p)=p$ and $f_{* p}=\phi$. Hence $f_{* p} X=Y$ and $f_{*}\left(\mathcal{H}_{p}\right)=\mathcal{H}_{p}$. Since $f_{*} A_{E} F=A_{f_{*} E} f_{*} F$ for every $E, F \in T_{p} M$, we see, by Theorem 2.4, that there is an isometry $\tilde{f}: B \rightarrow B$ such that $\tilde{f} \circ \pi=\pi \circ f$. Thus $\tilde{f}_{*} X^{\prime}=\tilde{f}_{*} \pi_{*} X=$ $\pi_{*} f_{*} X=\pi_{*} Y=Y^{\prime}$ and $\tilde{f}(x)=\tilde{f}(\pi(p))=\pi(f(p))=\pi(p)=x$.

Therefore $B$ is an isotropic semi-Riemannian manifold. This completes the proof of Theorem 3.3 .

If the metric on the base space is negative definite, the following lemma follows from Theorem 3.3.

Lemma 3.7. If $\pi: M \rightarrow B$ is a semi-Riemannian submersion with connected totally geodesic fibres from an $(n+r)$-dimensional semi-Riemannian manifold $M$ of index $r^{\prime}+n$ and of constant negative curvature onto an $n$-dimensional semi-Riemannian manifold $B$ of index $n$, then $r^{\prime}=r$.

Proof. By Theorem 3.3, we have $n=q_{1}\left(r^{\prime}+1\right)+q_{2}\left(r-r^{\prime}\right)=\left(q_{1}+q_{2}\right)(r+1)$ for some nonnegative integers $q_{1}$ and $q_{2}$. Hence $0=q_{1}\left(r-r^{\prime}\right)+q_{2}\left(r^{\prime}+1\right)$. Since the right hand side is the sum of two non-negative numbers, it follows that $q_{1}\left(r-r^{\prime}\right)=0$ and $q_{2}\left(r^{\prime}+1\right)=0$. Therefore $q_{2}=0$. This implies $r^{\prime}=r$.

Remark. Changing simultaneously the signs of metrics on the total space and on the base space, any semi-Riemannian submersion, under the assumptions of Lemma 3.7, becomes a Riemannian submersion with totally geodesic fibres from a sphere onto a Riemannian manifold. This case was completely classified by Escobales (see [Esc1]) and Ranjan (see [Ran1]).

Proposition 3.8. Let $\pi: M \rightarrow B$ be a semi-Riemannian submersion with connected totally geodesic fibres from a complete simply connected semi-Riemannian manifold $M$ onto a semi-Riemannian manifold $B$. Then $B$ is simply connected and complete.

Proof. If $M$ is geodesically complete, then so is $B$ (see [Bes or Ba-la]). Since $M$ is a complete semi-Riemannian manifold and the fibres are totally geodesic, any fibre is also geodesically complete. By a theorem in [Rec], it follows that the horizontal distribution $\mathcal{H}$ is an Ehresmann connection. Therefore, by [Ehr], we see that $\pi$ is a fibre bundle. So we obtain an exact homotopy sequence:

$$
\cdots \rightarrow \pi_{2}(M) \rightarrow \pi_{2}(B) \rightarrow \pi_{1}(\text { fibre }) \rightarrow \pi_{1}(M) \rightarrow \pi_{1}(B) \rightarrow 0
$$

Thus $\pi_{1}(B)=0$.

By Theorem 12.3.2 in [Wol], we know that any connected, simply connected isotropic semi-Riemannian manifold is isometric to one of the following semi-Riemannian manifolds: 
(i) $\mathbb{R}_{t}^{m}$ or the universal semi-Riemannian covering of the pseudo-hyperbolic space $H_{t}^{m}(c)$ with constant sectional curvature $c<0$, or of the pseudo-sphere $S_{t}^{m}(c)$ with constant sectional curvature $c>0$.

(ii) The complex pseudo-hyperbolic space $\mathbb{C} H_{t}^{m}(c)$ with constant holomorphic sectional curvature $c<0$, or the complex pseudo-projective space $\mathbb{C} P_{t}^{m}(c)$ with constant holomorphic sectional curvature $c>0$.

(iii) The quaternionic pseudo-hyperbolic space $\mathbb{H} H_{t}^{m}(c)$ with constant quaternionic sectional curvature $c<0$, or the quaternionic pseudo-projective space $\mathbb{H} P_{t}^{m}(c)$ with constant quaternionic sectional curvature $c>0$.

(iv) The Cayley pseudo-hyperbolic plane $\mathbb{C} a H_{t}^{2}(c)$ with Cayley sectional curvature $c<$ 0 , or the Cayley pseudo-projective plane $\mathbb{C} a P_{t}^{2}(c)$ with Cayley sectional curvature $c>0$.

Lemma 3.9. (a) If $B$ is a semi-Riemannian manifold isometric to one of the semiRiemannian manifolds $\mathbb{C} P_{t}^{m}(c), \mathbb{H} P_{t}^{m}(c), \mathbb{C} a P_{t}^{2}(c)(c>0)$, then the curvature tensor satisfies the inequality

$$
R^{\prime}\left(X^{\prime}, Y^{\prime}, X^{\prime}, Y^{\prime}\right) \geq \frac{c}{4}\left(g^{\prime}\left(X^{\prime}, X^{\prime}\right) g^{\prime}\left(Y^{\prime}, Y^{\prime}\right)-g^{\prime}\left(X^{\prime}, Y^{\prime}\right)^{2}\right)
$$

for each tangent vectors $X^{\prime}, Y^{\prime}$ of $B$.

(b) If $B$ is a semi-Riemannian manifold isometric to one of the semi-Riemannian manifolds $\mathbb{C} H_{t}^{m}(c), \mathbb{H} H_{t}^{m}(c), \mathbb{C} a H_{t}^{2}(c)(c<0)$, then the curvature tensor satisfies the inequality

$$
R^{\prime}\left(X^{\prime}, Y^{\prime}, X^{\prime}, Y^{\prime}\right) \leq \frac{c}{4}\left(g^{\prime}\left(X^{\prime}, X^{\prime}\right) g^{\prime}\left(Y^{\prime}, Y^{\prime}\right)-g^{\prime}\left(X^{\prime}, Y^{\prime}\right)^{2}\right)
$$

for each tangent vectors $X^{\prime}, Y^{\prime}$ of $B$.

Proof. For each tangent vectors $X^{\prime}, Y^{\prime}$ of $B$, we have the following formulas for the curvature tensors:

(i) If $B \in\left\{\mathbb{C} P_{t}^{m}(c), \mathbb{C} H_{t}^{m}(c)\right\}$ and $I_{0}$ is the natural complex structure on $B$, then

$$
R^{\prime}\left(X^{\prime}, Y^{\prime}, X^{\prime}, Y^{\prime}\right)=\frac{c}{4}\left(g^{\prime}\left(X^{\prime}, X^{\prime}\right) g^{\prime}\left(Y^{\prime}, Y^{\prime}\right)-g^{\prime}\left(X^{\prime}, Y^{\prime}\right)^{2}+3 g^{\prime}\left(X^{\prime}, I_{0} Y^{\prime}\right)^{2}\right) .
$$

(ii) If $B \in\left\{\mathbb{H} P_{t}^{m}(c), \mathbb{H} H_{t}^{m}(c)\right\}$ and $I_{0}, J_{0}, K_{0}$ are local almost complex structures which give rise to the quaternionic structure on $B$, then

$$
\begin{aligned}
R^{\prime}\left(X^{\prime}, Y^{\prime}, X^{\prime}, Y^{\prime}\right)= & (c / 4)\left(g^{\prime}\left(X^{\prime}, X^{\prime}\right) g^{\prime}\left(Y^{\prime}, Y^{\prime}\right)-g^{\prime}\left(X^{\prime}, Y^{\prime}\right)^{2}\right. \\
& \left.+3 g^{\prime}\left(X^{\prime}, I_{0} Y^{\prime}\right)^{2}+3 g^{\prime}\left(X^{\prime}, J_{0} Y^{\prime}\right)^{2}+3 g^{\prime}\left(X^{\prime}, K_{0} Y^{\prime}\right)^{2}\right)
\end{aligned}
$$

By these explicit formulas for curvature tensors, in all cases we obtain the inequalities (3.2) and (3.3).

First, we shall discuss the case of a base space with nonconstant curvature. 
Lemma 3.10. If $\pi: H_{s+r^{\prime}}^{n+r} \rightarrow B_{s}^{n}$ is a semi-Riemannian submersion with connected totally geodesic fibres from an $(n+r)$-dimensional pseudo-hyperbolic space $H_{s+r^{\prime}}^{n+r}$ of index $s+r^{\prime}>1$ onto an $n$-dimensional isotropic semi-Riemannian manifold $B_{s}^{n}$ of index $s$ with nonconstant curvature, then the induced metrics on the fibres are negative definite and $B$ is isometric to one of the following semi-Riemannian manifolds:

(i) $\mathbb{C} H_{t}^{m}, m>1$,

(ii) $\mathbb{H} H_{t}^{m}, m>1$,

(iii) $\mathbb{C} a H_{t}^{2}$.

Proof. Since $\operatorname{dim} \mathcal{H}=k(\operatorname{dim} \mathcal{V}+1)$ for some positive integer $k$, we get $\operatorname{dim} \mathcal{H} \geq \operatorname{dim} \mathcal{V}+1$. Let $X$ be a horizontal vector field along a fibre $\pi^{-1}(\pi(p))$ such that $g(X, X) \neq 0$ and $X$ is the horizontal lifting of some tangent vector of $B$.

First, we shall prove that

$$
\operatorname{dim} \mathcal{H}>\operatorname{dim} \mathcal{V}+1
$$

Suppose that $\operatorname{dim} \mathcal{H}=\operatorname{dim} \mathcal{V}+1$. Then $A_{X}: \mathcal{V} \rightarrow X^{\perp}=\{Y \in \mathcal{H} \mid g(X, Y)=0\}$ is bijective. For every $Y \in X^{\perp}$ we get $Y=A_{X} V$ for some vertical vector $V$. It follows that

$$
\begin{aligned}
g\left(A_{X} Y, A_{X} Y\right) & =g\left(A_{X} A_{X} V, A_{X} A_{X} V\right)=g(X, X)^{2} g(V, V), \\
g(Y, Y) & =g\left(A_{X} V, A_{X} V\right)=-g(X, X) g(V, V) .
\end{aligned}
$$

Thus $g\left(A_{X} Y, A_{X} Y\right)=-g(X, X) g(Y, Y)$ for every $Y \in X^{\perp}$. By O'Neill's equations, we have

$$
\begin{aligned}
R^{\prime}\left(\pi_{*} X, \pi_{*} Y, \pi_{*} X, \pi_{*} Y\right) & =-g(X, X) g(Y, Y)+g(X, Y)^{2}+3 g\left(A_{X} Y, A_{X} Y\right) \\
& =-4\left(g(X, X) g(Y, Y)-g(X, Y)^{2}\right)
\end{aligned}
$$

for every horizontal vector field $Y$ along $\pi^{-1}(\pi(p))$. Hence $B$ has constant curvature is a contradiction.

We established that $\operatorname{dim} \mathcal{H}>\operatorname{dim} \mathcal{V}+1$. So we can find a horizontal vector field $Z$ along the fibre $\pi^{-1}(\pi(p))$ such that $Z \in \operatorname{ker} A_{X}^{*}, g(X, Z)=0, g(Z, Z) \neq 0$ and $Z$ is the horizontal lifting of some $Z^{\prime} \in T_{\pi(p)} B$. We then have

$$
\begin{aligned}
R^{\prime}\left(\pi_{*} X, \pi_{*} Z, \pi_{*} X, \pi_{*} Z\right) & =-g(X, X) g(Z, Z)+g(X, Z)^{2}+3 g\left(A_{X} Z, A_{X} Z\right) \\
& =-g(X, X) g(Z, Z) .
\end{aligned}
$$

Since $B$ is a simply connected isotropic semi-Riemannian manifold with nonconstant curvature, we see that $B$ is isometric to one of the following semi-Riemannian manifolds:

(a) $\mathbb{C} P_{t}^{m}(c), \mathbb{H} P_{t}^{m}(c), \mathbb{C} a P_{t}^{2}(c)$, or

(b) $\mathbb{C} H_{t}^{m}(c), \mathbb{H} H_{t}^{m}(c), \mathbb{C} a H_{t}^{2}(c)$.

We shall prove that only the case (b) is possible.

First, we suppose that $B$ is isometric to one of the following semi-Riemannian manifolds:

$$
\mathbb{C} P_{t}^{m}(c), \mathbb{H} P_{t}^{m}(c), \mathbb{C} a P_{t}^{2}(c)(c>0) .
$$


By the inequality (3.2), we get

$$
\begin{aligned}
R^{\prime}\left(\pi_{*} X, \pi_{*} A_{X} V, \pi_{*} X, \pi_{*} A_{X} V\right) & =-4 g(X, X) g\left(A_{X} V, A_{X} V\right) \\
& =4 g(X, X)^{2} g(V, V) \geq-(c / 4) g(X, X)^{2} g(V, V) .
\end{aligned}
$$

Therefore

$$
g(V, V) \geq 0
$$

for every vertical vector $V$. Since $X$ and $Z$ are basic vector fields along $\pi^{-1}(\pi(p))$ with $g(X, Z)=0$ and $A_{X} Z=0$ along $\pi^{-1}(\pi(p))$, it follows from the relation (3.1) that $A_{Z} V \in$ ker $A_{X}^{*}$. On the other hand, by the inequality (3.2), we get

$$
\begin{aligned}
R^{\prime}\left(\pi_{*} X, \pi_{*} Z, \pi_{*} X, \pi_{*} Z\right) & =-g(X, X) g(Z, Z) \geq(c / 4) g(X, X) g(Z, Z), \\
R^{\prime}\left(\pi_{*} X, \pi_{*} A_{Z} V, \pi_{*} X, \pi_{*} A_{Z} V\right) & =-g(X, X) g\left(A_{Z} V, A_{Z} V\right) \geq(c / 4) g(X, X) g\left(A_{Z} V, A_{Z} V\right) .
\end{aligned}
$$

Hence $g(X, X) g(Z, Z) \leq 0$ and $g(X, X) g\left(A_{Z} V, A_{Z} V\right) \leq 0$. Thus

$$
0 \leq g(Z, Z) g\left(A_{Z} V, A_{Z} V\right)=-g(Z, Z)^{2} g(V, V) .
$$

So for any vertical vector $V$ we get

$$
g(V, V) \leq 0
$$

Since the induced metrics on fibres are nondegenerate, it is not possible to have both (3.6) and (3.7). So we obtain the required contradiction. It follows that $B$ is isometric to one of the following semi-Riemannian manifolds:

$$
\mathbb{C} H_{t}^{m}(c), \mathbb{H} H_{t}^{m}(c), \mathbb{C} a H_{t}^{2}(c) \quad(c<0) .
$$

We shall now prove that $c=-4$. Suppose $(c / 4)+1 \neq 0$. By the inequality (3.3), we get

$$
R^{\prime}\left(\pi_{*} X, \pi_{*} A_{Z} V, \pi_{*} X, \pi_{*} A_{Z} V\right)=-g(X, X) g\left(A_{Z} V, A_{Z} V\right) \leq(c / 4) g(X, X) g\left(A_{Z} V, A_{Z} V\right) .
$$

Hence

$$
((c / 4)+1)^{2} g(X, X)^{2} g(Z, Z) g\left(A_{Z} V, A_{Z} V\right) \geq 0,
$$

from which follows that $0 \leq g(Z, Z) g\left(A_{Z} V, A_{Z} V\right)=-g(Z, Z)^{2} g(V, V)$. Therefore $g(V, V) \leq$ 0 for every vertical vector field $V$. In particular, we have $g\left(A_{X} Y, A_{X} Y\right) \leq 0$, which implies

$$
R^{\prime}\left(\pi_{*} X, \pi_{*} Y, \pi_{*} X, \pi_{*} Y\right) \leq g(X, X) g(Y, Y)-g(X, Y)^{2}
$$

for every horizontal vectors $X$ and $Y$. We have the following cases:

Case (a) $0<\operatorname{index} B<\operatorname{dim} B$. We can choose vector fields $X^{\prime}, Y^{\prime}$ on $B$ such that $g^{\prime}\left(X^{\prime}, X^{\prime}\right) g^{\prime}\left(Y^{\prime}, Y^{\prime}\right)<0$ and that one of the following conditions is satisfied: 
(i) $Y^{\prime} \in\left\{X^{\prime}, I_{0} X^{\prime}\right\}^{\perp}$ if $B=\mathbb{C} H_{s}^{m}(c)$, where $I_{0}$ is the natural complex structure on $\mathbb{C} H_{s}^{m}(c)$,

(ii) $Y^{\prime} \in\left\{X^{\prime}, I_{0} X^{\prime}, J_{0} X^{\prime}, K_{0} X^{\prime}\right\}^{\perp}$ if $B=\mathbb{H} H_{s}^{m}(c)$, where $\left\{I_{0}, J_{0}, K_{0}\right\}$ are local almost complex structures which give rise to the quaternionic structure on $\mathbb{H} H_{s}^{m}(c)$, or

Let $X, Y$ be the horizontal liftings of $X^{\prime}, Y^{\prime}$. The inequality (3.10) then implies

$$
\frac{c}{4} g(X, X) g(Y, Y) \leq-g(X, X) g(Y, Y) .
$$

Hence $((c / 4)+1) g(X, X) g(Y, Y) \leq 0$. Therefore $(c / 4)+1>0$. On the other hand, we can choose horizontal vector fields $X, Z$ such that $g(X, Z)=0, Z \in \operatorname{ker} A_{X}^{*}$ and $g(X, X) g(Z, Z)<0$, because $0<\operatorname{index} B<\operatorname{dim} B$. Then the inequality (3.8) becomes $(c / 4)+1<0$. So we get a contradiction.

Case (b) index $B \in\{0, \operatorname{dim} B\}$. Similarly, we can choose vector fields $X^{\prime}, Y^{\prime}$ on $B$ such that $g^{\prime}\left(X^{\prime}, Y^{\prime}\right)=0$ and $R^{\prime}\left(X^{\prime}, Y^{\prime}, X^{\prime}, Y^{\prime}\right)=(c / 4) g^{\prime}\left(X^{\prime}, X^{\prime}\right) g^{\prime}\left(Y^{\prime}, Y^{\prime}\right)$. The inequality (3.10) then implies $((c / 4)+1) g^{\prime}\left(X^{\prime}, X^{\prime}\right) g^{\prime}\left(Y^{\prime}, Y^{\prime}\right) \leq 0$. By the hypothesis of Case (b), we get $(c / 4)+1 \leq 0$. On the other hand, the inequality (3.8) becomes $(c / 4)+1>0$. So we get a contradiction.

We have proved $c=-4$. The inequality (3.3) then becomes

$$
R^{\prime}\left(X^{\prime}, Y^{\prime}, X^{\prime}, Y^{\prime}\right) \leq-g^{\prime}\left(X^{\prime}, X^{\prime}\right) g^{\prime}\left(Y^{\prime}, Y^{\prime}\right)+g^{\prime}\left(X^{\prime}, Y^{\prime}\right)^{2}
$$

for tangent vector fields $X^{\prime}, Y^{\prime}$ on $B$. Then we have

$$
R^{\prime}\left(\pi_{*} X, \pi_{*} A_{X} V, \pi_{*} X, \pi_{*} A_{X} V\right)=-4 g(X, X) g\left(A_{X} V, A_{X} V\right) \leq-g(X, X) g\left(A_{X} V, A_{X} V\right)
$$

for a vertical vector field $V$ and for a horizontal vector field $X$. Hence

$$
0 \leq g(X, X) g\left(A_{X} V, A_{X} V\right)=-g(X, X)^{2} g(V, V) .
$$

Therefore the induced metrics on fibres are negative definite.

By Lemma 3.10, we deduce the following proposition.

Proposition 3.11. If $\pi: H_{s+r^{\prime}}^{n+r} \rightarrow B_{s}^{n}$ is a semi-Riemannian submersion with connected totally geodesic fibres from an $(n+r)$-dimensional pseudo-hyperbolic space $H_{s+r^{\prime}}^{n+r}$ of index $s+r^{\prime}$ onto an n-dimensional isotropic semi-Riemannian manifold $B_{s}^{n}$ of index $s$ with nonconstant curvature, and if the fibres are negatively definite then one of the following holds:

(1) $n=2 m>2, s=2 t, r=r^{\prime}=1$ for some non-negative integers $m$, $t$, and $B_{s}^{n}$ is isometric to $\mathbb{C} H_{t}^{m}$.

(2) $n=4 m>4, s=4 t, r=r^{\prime}=3$ for some non-negative integers $m$, $t$, and $B_{s}^{n}$ is isometric to $\mathbb{H} H_{t}^{m}$.

(3) $n=16, s \in\{0,8,16\}, r=r^{\prime}=7$, and $B_{s}^{n}$ is isometric to $\mathbb{C} a H_{s / 8}^{2}$. 
Proof. First, we shall discuss the case $s+r^{\prime}>1$. By Lemma 3.10, $B$ is isometric to one of the semi-Riemannian manifolds $\mathbb{C} H_{t}^{m}, \mathbb{H} H_{t}^{m}, \mathbb{C} a H_{t}^{2}$ for some $m>1$.

Let $x \in B$ and let $X^{\prime} \in T_{x} B$ such that $g^{\prime}\left(X^{\prime}, X^{\prime}\right) \neq 0$, and let $\mathcal{F}_{X^{\prime}}$ be the subspace in $T_{x} B$ given by

$$
\mathcal{F}_{X^{\prime}}=\left\{Y^{\prime} \in T_{x} B \mid R^{\prime}\left(X^{\prime}, Y^{\prime}\right) X^{\prime}=-g^{\prime}\left(X^{\prime}, Y^{\prime}\right) X^{\prime}+g^{\prime}\left(X^{\prime}, X^{\prime}\right) Y^{\prime}\right\} .
$$

Let $p \in \pi^{-1}(x)$ and let $X$ be the horizontal lifting vector at $p$ of $X^{\prime}$. By O'Neill's equations, we have $R^{\prime}\left(\pi_{*} X, \pi_{*} Y, \pi_{*} X, \pi_{*} Z\right)=R(X, Y, X, Z)+3 g\left(A_{X}^{*} Y, A_{X}^{*} Z\right)$ for horizontal vectors $Y, Z$. Since $A_{X}^{*}: \mathcal{H}_{p} \rightarrow \mathcal{V}_{p}$ is surjective and since the induced metrics on fibres are nondegenerate, we get $Y \in \operatorname{ker} A_{X}^{*}$ if and only if $\pi_{*} Y \in \mathcal{F}_{X^{\prime}}$. Thus

$$
\operatorname{dim} \operatorname{ker} A_{X}^{*}=\operatorname{dim} \mathcal{H}-\operatorname{dim} \mathcal{V}=\operatorname{dim} \mathcal{F}_{X^{\prime}}
$$

We have the following possibilities:

(1) $B_{s}^{n}$ is isometric to $\mathbb{C} H_{t}^{m}$. So $n=2 m, s=2 t$. From the geometry of the complex pseudo-hyperbolic space (see relation $(\underline{3.4})$ ), we get $\operatorname{dim} \mathcal{F}_{X^{\prime}}=\operatorname{dim} \mathcal{H}-1$. It follows that $r=r^{\prime}=\operatorname{dim} \mathcal{V}=1$.

(2) $B_{s}^{n}$ is isometric to $\mathbb{H} H_{t}^{m}$. So $n=4 m, s=4 t$. From the geometry of the quaternionic pseudo-hyperbolic space (see relation $(3.5)$ ), we get $\operatorname{dim} \mathcal{F}_{X^{\prime}}=\operatorname{dim} \mathcal{H}-3$. It follows that $r=r^{\prime}=\operatorname{dim} \mathcal{V}=3$.

(3) $B_{s}^{n}$ is isometric to the Cayley pseudo-hyperbolic plane $\mathbb{C} a H_{t}^{2}$. So $n=16, s \in\{0,8,16\}$. From the geometry of the Cayley pseudo-hyperbolic plane, we obtain $\operatorname{dim} \mathcal{F}_{X^{\prime}}=$ $\operatorname{dim} \mathcal{H}-7$. Hence $r=r^{\prime}=\operatorname{dim} \mathcal{V}=7$.

Now, we discuss the remaining case $s+r^{\prime}=1$. From $s+r^{\prime}=1$, we have either

(i) $s=0, r^{\prime}=1$, or

(ii) $s=1, r^{\prime}=0$.

If $s=0, r^{\prime}=1$, then $\pi: H_{1}^{n+r} \rightarrow B^{n}$ is a semi-Riemannian submersion with totally geodesic fibres from an anti-de Sitter space onto a Riemannian manifold. In this case, investigated by Magid in $\mathrm{Mag}$, it follows that $B$ is isometric to the complex hyperbolic space $\mathbb{C} H^{m}$ and $r=r^{\prime}=1$.

For $s=1, r^{\prime}=0$, we get, by Theorem 3.3, $1=q_{1}+q_{2} r \geq q_{1}+q_{2}$ with $q_{1}+q_{2}=$ $k=n /(r+1)$. Thus $q_{1}+q_{2}=1$. It follows that $n=r+1$. Hence $A_{X}: \mathcal{V} \rightarrow X^{\perp}$ is bijective. Since $R^{\prime}\left(\pi_{*} X, \pi_{*} A_{X} V, \pi_{*} X, \pi_{*} A_{X} V\right)=-4 g(X, X) g\left(A_{X} V, A_{X} V\right)$, we see that $B$ has constant curvature -4 , which contradicts our assumption of nonconstant curvature of the base space.

We shall now discuss the case where the base space is of constant curvature.

Proposition 3.12. If $\pi: H_{s+r^{\prime}}^{n+r} \rightarrow B_{s}^{n}$ is a semi-Riemannian submersion with connected totally geodesic fibres from an $(n+r)$-dimensional pseudo-hyperbolic space of index $s+r^{\prime}$ 
onto an $n$-dimensional semi-Riemannian manifold of index s with constant curvature, and if the fibres are negatively definite, then one of the following holds:

(1) $n=s=2^{t}, r=r^{\prime}=n-1, B$ is isometric to $H_{2^{t}}^{2^{t}}(-4)$ and $t \in\{1,2,3\}$.

(2) $n=2^{t}, s=0, r=r^{\prime}=n-1, B$ is isometric to $H^{2^{t}}(-4)$ and $t \in\{1,2,3\}$.

Proof. Since $B$ has constant curvature, the curvature of $B$ is -4 and $n=r+1$. By Theorem 3.3, $s=q_{1}\left(r^{\prime}+1\right)+q_{2}\left(r-r^{\prime}\right)=q_{1}(r+1)$ and $q_{1}+q_{2}=n /(r+1)=1$. Then either $q_{1}=0$ or $q_{1}=1$. If $q_{1}=0$, then $s=0$. If $q_{1}=1$ then $s=r+1=n$. Summarizing, we have $\operatorname{index}(B) \in\{0, \operatorname{dim} B\}$.

If $\operatorname{index}(B)=\operatorname{dim} B$, then, by Lemma 3.7, we obtain $r=r^{\prime}$. Hence, by [Ran1], we have (1).

If $\operatorname{index}(B)=0$, then, by Ba-Ia], we have (2).

The idea of the proof in [Ran1] and [Ba-Ia] is to see that the tangent bundle of any fibre is trivial and that fibres are diffeomorphic to spheres, and then to apply a well-known result of Adams which claims that the spheres of dimensions 1, 3 and 7 are the only spheres with trivial tangent bundle.

The next theorems solve the equivalence problem of semi-Riemannian submersions from real and complex pseudo-hyperbolic spaces.

Theorem 3.13. If $\pi_{1}, \pi_{2}: H_{s+r^{\prime}}^{n+r} \rightarrow B_{s}^{n}$ are two semi-Riemannian submersions with connected totally geodesic fibres from a pseudo-hyperbolic space of index $s+r^{\prime}>1$, if the fibres are negative definite, and if the dimension of the fibres is $r \in\{1,3\}$, then $\pi_{1}$ and $\pi_{2}$ are equivalent.

Proof. Let $p, q \in H_{s+r^{\prime}}^{n+r}$. Let

$$
\begin{aligned}
& \mathcal{L}=\left\{L_{0}, A_{1 L_{0}} v_{1}, \ldots, A_{1 L_{0}} v_{r}, \ldots, L_{k-1}, A_{1 L_{k-1}} v_{1}, \ldots, A_{1 L_{k-1}} v_{r}\right\} \\
& \mathcal{L}^{\prime}=\left\{L_{0}^{\prime}, A_{2 L_{0}^{\prime}} v_{1}^{\prime}, \ldots, A_{2 L_{0}^{\prime}} v_{r}^{\prime}, \ldots, L_{k-1}^{\prime}, A_{2 L_{k-1}^{\prime}} v_{1}^{\prime}, \ldots, A_{2 L_{k-1}^{\prime}} v_{r}^{\prime}\right\}
\end{aligned}
$$

be two orthonormal bases of $\mathcal{H}_{1}$ along $\pi_{1}^{-1}\left(\pi_{1}(p)\right)$ and of $\mathcal{H}_{2}$ along $\pi_{2}^{-1}\left(\pi_{2}(q)\right)$ constructed as in the proof of Theorem 3.3 such that $g_{p}\left(L_{\alpha}, L_{\beta}\right)=g_{q}\left(L_{\alpha}^{\prime}, L_{\beta}^{\prime}\right)=\varepsilon_{\alpha} \delta_{\alpha \beta}$ for $\alpha, \beta \in$ $\{0, \ldots, k-1\}, g_{p}\left(v_{i}, v_{j}\right)=g_{q}\left(v_{i}^{\prime}, v_{j}^{\prime}\right)=\varepsilon_{i} \delta_{i j}$ for $i, j \in\{1, \ldots, r\}$ and for $r=3, v_{3 p}=$ $\left(\hat{\nabla}_{v_{1}} v_{2}\right)(p)$ and $v_{3 q}^{\prime}=\left(\hat{\nabla}_{v_{1}^{\prime}} v_{2}^{\prime}\right)(q)$.

Let $\phi: T_{p} H_{s+r^{\prime}}^{n+r} \rightarrow T_{q} H_{s+r^{\prime}}^{n+r}$ be the linear map given by $\phi\left(L_{\alpha}\right)=L_{\alpha}^{\prime}, \phi\left(A_{1 L_{\alpha}} v_{i}\right)=A_{2 L_{\alpha}^{\prime}} v_{i}^{\prime}$, $\phi\left(v_{i}\right)=v_{i}^{\prime}$ for every $\alpha$ and $i$. In a manner similar to the proof of Theorem 3.3, we obtain $\phi\left(A_{1 E} F\right)=A_{2 \phi(E)} \phi(F)$ for every $E, F \in T_{p} H_{s+r^{\prime}}^{n+r}$. By Corollary 2.3.14 in [Wol], $\phi$ extends to an isometry on $H_{s+r^{\prime}}^{n+r}$, denoted by $f: H_{s+r^{\prime}}^{n+r} \rightarrow H_{s+r^{\prime}}^{n+r}$, satisfying $f(p)=q$ and $f_{* p}=\phi$. From Theorem 2.4 it follows that $f$ induces an isometry $\tilde{f}$ on $B$, such that $\tilde{f} \circ \pi=\pi \circ f$. Hence $\pi_{1}$ and $\pi_{2}$ are equivalent. 
Theorem 3.14. If $\pi_{1}, \pi_{2}: \mathbb{C} H_{2 s+1}^{2 n+1} \rightarrow \mathbb{H} H_{s}^{n}$ are two semi-Riemannian submersions with connected complex totally geodesic fibres from a complex pseudo-hyperbolic space, and if the fibres are negative definite, then $\pi_{1}$ and $\pi_{2}$ are equivalent.

Proof. Let $\theta: H_{4 s+3}^{4 n+3} \rightarrow \mathbb{C} H_{2 s+1}^{2 n+1}$ be the canonical semi-Riemannian submersion. By Theorem 2.5 in [Esc2], we see that $\tilde{\pi}_{1}=\pi_{1} \circ \theta: H_{4 s+3}^{4 n+3} \rightarrow \mathbb{H} H_{s}^{n}$ and $\tilde{\pi}_{2}=\pi_{2} \circ \theta: H_{4 s+3}^{4 n+3} \rightarrow$ $\mathbb{H} H_{s}^{n}$ are semi-Riemannian submersions with totally geodesic fibres. We denote by $\tilde{A}_{1}$, $\tilde{A}_{2}, A_{1}, A_{2}, A$ O'Neill's integrability tensors of $\tilde{\pi}_{1}, \tilde{\pi}_{2}, \pi_{1}, \pi_{2}, \theta$, respectively. In order to reduce the proof of the equivalence theorem of semi-Riemannian submersions from a complex pseudo-hyperbolic space to that from a pseudo-hyperbolic space, we need to establish relations among the integrability tensors $\tilde{A}_{1}, A_{1}, A$.

First, we prove that $\theta_{*} \tilde{A}_{1 X} Y=A_{1 \theta_{*} X} \theta_{*} Y$ for $\tilde{\pi}_{1}$-basic vector fields $X$ and $Y$. Let $p \in H_{4 s+3}^{4 n+3}$. Let $w_{1}^{\prime}, w_{2}^{\prime}$ be two orthonormal $\pi_{1}$-vertical vectors in $T_{\theta(p)} \mathbb{C} H_{2 s+1}^{2 n+1}$ and let $w_{1}$, $w_{2}$ be the $\theta$-horizontal liftings at $p$ of $w_{1}^{\prime}, w_{2}^{\prime}$, respectively. Let $w_{3}$ be a unit $\theta$-vertical vector in $T_{p} H_{4 s+3}^{4 n+3}$. Then $\left\{w_{1}, w_{2}, w_{3}\right\}$ gives an orthonormal basis of $\tilde{\mathcal{V}}_{1 p}$. Since the induced metrics on the fibres of $\tilde{\pi}_{1}$ are negative definite, we have

$$
\tilde{A}_{1 X} Y=-g\left(\nabla_{X} Y, w_{1}\right) w_{1}-g\left(\nabla_{X} Y, w_{2}\right) w_{2}-g\left(\nabla_{X} Y, w_{3}\right) w_{3} .
$$

Thus

$$
\theta_{*} \tilde{A}_{1 X} Y=-g^{\prime}\left(\nabla_{\theta_{*} X}^{\prime} \theta_{*} Y, \theta_{*} w_{1}\right) \theta_{*} w_{1}-g^{\prime}\left(\nabla_{\theta_{*} X}^{\prime} \theta_{*} Y, \theta_{*} w_{2}\right) \theta_{*} w_{2}=A_{1 \theta_{*} X} \theta_{*} Y
$$

for $\tilde{\pi}_{1}$-basic vector fields $X$ and $Y$, where $g^{\prime}$ denotes the metric on $\mathbb{C} H_{2 s+1}^{2 n+1}$ and $\nabla^{\prime}$ is the Levi-Civita connection of $g^{\prime}$.

Let $X$ be the $\tilde{\pi}_{1}$-horizontal lifting along the fibre $\tilde{\pi}_{1}^{-1}\left(\tilde{\pi}_{1}(p)\right)$ of some unit vector in $T_{\tilde{\pi}_{1}(p)} \mathbb{H} H_{s}^{n}$. Let $Y_{1}, Y_{2}, Y_{3}$ be the $\tilde{\pi}_{1}$-horizontal liftings along the fibre $\tilde{\pi}_{1}^{-1}\left(\tilde{\pi}_{1}(p)\right)$ of $\tilde{\pi}_{1 *} \tilde{A}_{1 X} w_{1}, \tilde{\pi}_{1 *} \tilde{A}_{1 X} w_{2}, \tilde{\pi}_{1 *} \tilde{A}_{1 X} w_{3}$, respectively. Let $v_{i}=\tilde{A}_{1 X} Y_{i}$ for $i \in\{1,2,3\}$. As in Theorem 3.3, we choose $w_{3}=g(X, X)^{-1}\left(\nabla_{v_{1}} v_{2}\right)(p)$, which implies that $v_{3}=\nabla_{v_{1}} v_{2}$ (see Lemma 3.6).

We remark that $v_{3}=\tilde{A}_{1 X} Y_{3}$ is a $\theta$-vertical vector field along the fibre $\theta^{-1}(\theta(p))$. Indeed, we have

$$
\begin{aligned}
\theta_{*}\left(\tilde{A}_{1 X} Y_{3}\left(p^{\prime}\right)\right) & =\left(A_{1 \theta_{*} X} \theta_{*} Y_{3}\right)\left(\theta\left(p^{\prime}\right)\right)=\left(A_{1 \theta_{*} X} \theta_{*} Y_{3}\right)(\theta(p))=\theta_{*}\left(\tilde{A}_{1 X} Y_{3}(p)\right) \\
& =\theta_{*}\left(A_{1 X} A_{1 X} w_{3}\right)=g(X, X) \theta_{*} w_{3}=0
\end{aligned}
$$

for any $p^{\prime} \in \theta^{-1}(\theta(p))$.

Since $v_{1}, v_{2}$ are orthogonal to the vertical vector field $v_{3}$ along $\theta^{-1}(\theta(p))$, we see that $v_{1}, v_{2}$ are $\theta$-horizontal. Since $\theta_{*}\left(\tilde{A}_{1 X} Y_{i}\left(p^{\prime}\right)\right)=\left(A_{1 \theta_{*} X} \theta_{*} Y_{i}\right)\left(\theta\left(p^{\prime}\right)\right)$ for $p^{\prime} \in \theta^{-1}(\theta(p))$ and for $i \in\{1,2\}$, we obtain that $v_{1}, v_{2}$ are $\theta$-basic vector fields along $\theta^{-1}(\theta(p))$. Thus $h \nabla_{v_{3}} v_{1}=A_{v_{1}} v_{3}$ along $\theta^{-1}(\theta(p))$. Here $h$ and $v$ denote the $\theta$-horizontal and $\theta$-vertical projections, respectively. We also obtain that $v \nabla_{v_{3}} v_{1}=-g\left(\nabla_{v_{3}} v_{1}, v_{3}\right) v_{3}=0$. Therefore, $A_{v_{1}} v_{3}=\nabla_{v_{3}} v_{1}=v_{2}$ along $\theta^{-1}(\theta(p))$. 
We shall prove that $\tilde{A}_{1 X} v_{3}=A_{X} v_{3}$ along $\theta^{-1}(\theta(p))$ for every $\tilde{\pi}_{1}$-basic vector field $X$ along $\tilde{\pi}_{1}^{-1}\left(\tilde{\pi}_{1}(p)\right)$. We first obtain along $\theta^{-1}(\theta(p))$ that

$$
\begin{aligned}
\tilde{A}_{1 X} v_{3} & =\nabla_{X} v_{3}+g\left(\nabla_{X} v_{3}, v_{1}\right) v_{1}+g\left(\nabla_{X} v_{3}, v_{2}\right) v_{2}+g\left(\nabla_{X} v_{3}, v_{3}\right) v_{3}, \\
g\left(\nabla_{X} v_{3}, v_{1}\right) & =g\left(A_{X} v_{3}, v_{1}\right)=-g\left(v_{3}, A_{X} v_{1}\right)=g\left(v_{3}, A_{v_{1}} X\right)=-g\left(A_{v_{1}} v_{3}, X\right) \\
& =-g\left(v_{2}, X\right)=0
\end{aligned}
$$

for a $\tilde{\pi}_{1}$-basic vector field $X$ along $\tilde{\pi}_{1}^{-1}\left(\tilde{\pi}_{1}(p)\right)$. Analogously, we get $g\left(\nabla_{X} v_{3}, v_{2}\right)=0$. Thus

$$
\tilde{A}_{1 X} v_{3}=\nabla_{X} v_{3}+g\left(\nabla_{X} v_{3}, v_{3}\right) v_{3}=A_{X} v_{3}
$$

along $\theta^{-1}(\theta(p))$ for every $\tilde{\pi}_{1}$-basic vector field $X$ along $\tilde{\pi}_{1}^{-1}\left(\tilde{\pi}_{1}(p)\right)$.

Let $\tilde{\mathcal{L}}=\left\{L_{0}=X, \tilde{A}_{1 L_{0}} v_{1}, \tilde{A}_{1 L_{0}} v_{2}, \tilde{A}_{1 L_{0}} v_{3}, \ldots, L_{n-1}, \tilde{A}_{1 L_{n-1}} v_{1}, \tilde{A}_{1 L_{n-1}} v_{2}, \tilde{A}_{1 L_{n-1}} v_{3}\right\}$ be an orthonormal basis of $\tilde{\mathcal{H}}_{1}$ along the fibre $\tilde{\pi}_{1}^{-1}\left(\tilde{\pi}_{1}(p)\right)$ constructed as in Theorem 3.3 , for the semi-Riemannian submersion $\tilde{\pi}_{1}$. From the proof of Theorem 3.3, we have

$$
g\left(\tilde{A}_{1 \tilde{A}_{1 L_{j}} v_{1}} v_{3}, \tilde{A}_{1 L_{l}} v_{2}\right)=0
$$

for $j \neq l$, and

$$
g\left(\tilde{A}_{1 \tilde{A}_{1 L_{j} v_{1}}} v_{3}, L_{t}\right)=0
$$

for $0 \leq j, t \leq n-1$. We then obtain along $\tilde{\pi}_{1}^{-1}\left(\tilde{\pi}_{1}(p)\right)$ that

$$
\begin{aligned}
g\left(\tilde{A}_{\left.1 \tilde{A}_{1 L_{j} v_{1}} v_{3}, \tilde{A}_{1 L_{j}} v_{2}\right)}=-g\left(v_{3}, \tilde{A}_{1 \tilde{A}_{1 L_{j} v_{1}}} \tilde{A}_{1 L_{j}} v_{2}\right)\right. \\
=-g\left(v_{3}, \nabla_{v_{1}} v_{2}\right) g\left(L_{j}, L_{j}\right) \\
=-g\left(v_{3}, v_{3}\right) g\left(L_{j}, L_{j}\right)=-g\left(v_{2}, v_{2}\right) g\left(L_{j}, L_{j}\right) \\
=g\left(\tilde{A}_{1 L_{j}} v_{2}, \tilde{A}_{1 L_{j}} v_{2}\right),
\end{aligned}
$$

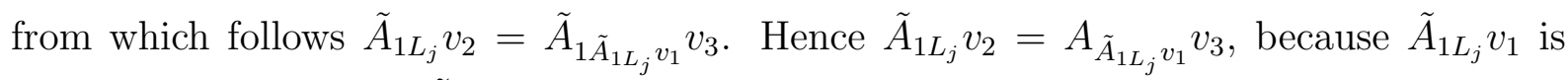
$\tilde{\pi}_{1}$-basic. We also have $\tilde{A}_{1 L_{j}} v_{3}=A_{L_{j}} v_{3}$.

Let $\mathcal{L}=\tilde{\mathcal{L}} \cup\left\{v_{1}, v_{2}\right\}$. Summarizing all the above, we obtain that

$$
\mathcal{L}=\left\{L_{0}, A_{L_{0}} v_{3}, \tilde{A}_{1 L_{0}} v_{1}, A_{\tilde{A}_{1 L_{0}} v_{1}} v_{3}, \ldots, L_{n-1}, A_{L_{n-1}} v_{3}, \tilde{A}_{L_{n-1}} v_{1}, A_{\tilde{A}_{1 L_{n-1}} v_{1}} v_{3}, v_{1}, A_{v_{1}} v_{3}\right\}
$$

is an orthonormal basis of the $\theta$-horizontal space $\mathcal{H}$ along the fibre $\theta^{-1}(\theta(p))$ and $\mathcal{L}$ satisfies all conditions imposed in the construction of the basis $\mathcal{L}$ in the proof of Theorem 3.3. We notice that $v_{3}=A_{X} Y_{3}$ along $\theta^{-1}(\theta(p))$, and that along $\theta^{-1}(\theta(p)), Y_{3}$ is equal to the $\theta$-horizontal lifting of $\theta_{*} A_{X} w_{3}$.

Let $q \in H_{4 s+3}^{4 n+3}$. Let

$$
\tilde{\mathcal{L}}^{\prime}=\left\{L_{0}^{\prime}, \tilde{A}_{2 L_{0}^{\prime}} v_{1}^{\prime}, \tilde{A}_{2 L_{0}^{\prime}} v_{2}^{\prime}, \tilde{A}_{2 L_{0}^{\prime}} v_{3}^{\prime}, \ldots, L_{n-1}^{\prime}, \tilde{A}_{2 L_{n-1}^{\prime}} v_{1}^{\prime}, \tilde{A}_{2 L_{n-1}^{\prime}} v_{2}^{\prime}, \tilde{A}_{2 L_{n-1}^{\prime}} v_{3}^{\prime}\right\}
$$

be an orthonormal basis of $\tilde{\mathcal{H}}_{2}$ along $\tilde{\pi}_{2}^{-1}\left(\tilde{\pi}_{2}(q)\right)$ constructed in the same way as $\tilde{\mathcal{L}}$, but for the semi-Riemannian submersion $\tilde{\pi}_{2}$ (see the proof of Theorem 3.3), in such a way that $g_{p}\left(L_{\alpha}, L_{\beta}\right)=g_{q}\left(L_{\alpha}^{\prime}, L_{\beta}^{\prime}\right)$ for $0 \leq \alpha, \beta \leq n-1, g_{p}\left(v_{i}, v_{j}\right)=g_{q}\left(v_{i}^{\prime}, v_{j}^{\prime}\right)$ for $1 \leq i, j \leq 3$, and 
$v_{3}^{\prime}(q)=\left(\nabla_{v_{1}^{\prime}} v_{2}^{\prime}\right)(q)$. Let $\phi: T_{p} H_{4 s+3}^{4 n+3} \rightarrow T_{q} H_{4 s+3}^{4 n+3}$ be the linear map given by $\phi\left(v_{i}\right)=v_{i}^{\prime}$, $\phi\left(\tilde{A}_{1 L_{\alpha}} v_{i}\right)=\tilde{A}_{2 L_{\alpha}^{\prime}} v_{i}^{\prime}$ for $0 \leq \alpha \leq n-1$ and for $1 \leq i \leq 3$.

By Corollary 2.3.14 in [Wol], $\phi$ extends to an isometry $f: H_{4 s+3}^{4 n+3} \rightarrow H_{4 s+3}^{4 n+3}$ such that $f(p)=q$ and $f_{* p}=\phi$. By the proof of Theorem 3.3. we have $f_{*} \tilde{A}_{1 E} F=\tilde{A}_{2 f_{*} E} f_{*} F$ for every $E, F \in T_{p} H_{4 s+3}^{4 n+3}$. By the proof of Theorem 3.13 and by Theorem $2.4, f$ induces an isometry on $\mathbb{C} H_{2 s+1}^{2 n+1}$, denoted by $\tilde{f}: \mathbb{C} H_{2 s+1}^{2 n+1} \rightarrow \mathbb{C} H_{2 s+1}^{2 n+1}$, such that $\theta \circ f=\tilde{f} \circ \theta$. Since the $\pi_{1}$-vertical space at $\theta(p)$ is spanned by $\left\{\theta_{*} v_{1}, \theta_{*} v_{2}\right\}$, since the $\pi_{2}$-vertical space at $\theta(q)$ is spanned by $\left\{\theta_{*} v_{1}^{\prime}, \theta_{*} v_{2}^{\prime}\right\}$, and since $\tilde{f}_{*}\left(\theta_{*} v_{i}\right)=\theta_{*} v_{i}^{\prime}$, for $i \in\{1,2\}$, we see that $\tilde{f}_{*}$ maps the $\pi_{1}$-vertical space at $\theta(p)$ into the $\pi_{2}$-vertical space at $\theta(q)$. For $\tilde{\pi}_{1}$-horizontal vectors $X$ and $Y$ we obtain

$$
\begin{aligned}
\tilde{f}_{*} A_{1 \theta_{*} X} \theta_{*} Y & =\tilde{f}_{*} \theta_{*} \tilde{A}_{1 X} Y=\theta_{*} f_{*} \tilde{A}_{1 X} Y \\
& =\theta_{*} \tilde{A}_{2 f_{*} X} f_{*} Y=A_{2 \theta_{*} f_{*} X} \theta_{*} f_{*} Y \\
& =A_{2 \tilde{f}_{*}\left(\theta_{*} X\right)} \tilde{f}_{*}\left(\theta_{*} Y\right) .
\end{aligned}
$$

Therefore, by Theorem 2.4, we see that $\pi_{1}$ and $\pi_{2}$ are equivalent.

Remark. We notice that our equivalence theorems can be applied, in particular, to Riemannian submersions from a sphere with totally geodesic fibres of dimension less than or equal to 3, and for Riemannian submersions with complex totally geodesic fibres from a complex projective space. Unlike those in [Esc1, [Esc2], [Ran1, our proofs of the equivalence theorems are intrinsic, we do not need to assume the existence of any specific structure on the base space, such as complex or quaternionic one. In Theorem 3.14, we need to assume only that the fibres are 2-dimensional and that the induced metrics on fibres are negative definite.

Summarizing all results above, we now prove the main theorems.

Proof of Theorem 1.1. If $s+r^{\prime}>1$, then $H_{s+r^{\prime}}^{n+r}$ is simply connected and hence, by Theorem 3.3, $B$ is an isotropic semi-Riemannian manifold and $r \in\{1,3\}$. By Propositions 3.11 and 3.12 , we see that the base space of the semi-Riemannian submersion is isometric to a complex pseudo-hyperbolic space if the dimension of fibres is one, or to a quaternionic pseudo-hyperbolic space if the dimension of fibres is 3 . In Theorem 3.13 we solved the equivalence problem. The existence problem is solved by the explicit construction given in the preliminaries (see Examples 1 and 2).

If $s+r^{\prime}=1$, then either (i) $s=1, r^{\prime}=0$, or (ii) $s=0, r^{\prime}=1$. Since the fibres are assumed to be negative definite, (i) cannot occur.

(ii) If $s=0, r^{\prime}=1$, then $\pi$ is a semi-Riemannian submersion from an anti-de Sitter space onto a Riemannian manifold. By $\mathrm{Mag}, \pi$ is equivalent to the canonical submersion $\pi: H_{1}^{2 m+1} \rightarrow \mathbb{C} H^{m}$. This falls in the case (a). 
Proof of Theorem 1.2. If the dimension of the fibres is less than or equal to 3, then, by Theorem 1.1, $\pi$ is equivalent to the canonical semi-Riemannian submersions:

(a) $H_{2 t+1}^{2 m+1} \rightarrow \mathbb{C} H_{t}^{m}, 0 \leq t \leq m$, or

(b) $H_{4 t+3}^{4 m+3} \rightarrow \mathbb{H} H_{t}^{m}, 0 \leq t \leq m$.

Now we assume that the dimension of the fibres is greater than or equal to 4 .

(A) If we assume that the dimension of the fibres is greater than or equal to 4 and $B$ is an isotropic semi-Riemannian manifold with non-constant curvature, then, by Proposition [3.11, $B$ is isometric to $\mathbb{C} a H_{t}^{2}, t \in\{0,1,2\}$, and the dimension of the fibres is $r=r^{\prime}=7$. By Proposition 2.7, there are no such semi-Riemannian submersions with base space $\mathbb{C} a H_{t}^{2}$. Therefore, the assumptions (A) and $r \geq 4$ imply that $B$ has constant curvature, and hence, by Proposition 3.12 , we obtain $s=\operatorname{index}(B) \in\{0, \operatorname{dim}(B)\}$.

(B) If $\operatorname{index}(B)=0$ and $r \geq 4$, then, by [Ba-Ia], the semi-Riemannian submersion $\pi$ is equivalent to the canonical semi-Riemannian submersion $H_{7}^{15} \rightarrow H^{8}(-4)$. If index $(B)=$ $\operatorname{dim}(B)$, then, by Lemma 3.7, we get $r^{\prime}=r$. By changing the signs of the metrics on the base and on the total space, $\pi$ becomes a Riemannian submersion with connected totally geodesic fibres from a sphere onto a Riemannian manifold. So, by [Esc1] and [Ran1], one obtains the conclusion.

Proof of Theorem 1.3. Let $\theta: H_{2 s+1}^{2 n+1} \rightarrow \mathbb{C} H_{s}^{n}$ be the canonical semi-Riemannian submersion. By Theorem 2.5 in [Esc2], one obtains that $\pi \circ \theta: H_{2 s+1}^{2 n+1} \rightarrow B$ is a semi-Riemannian submersion with connected totally geodesic fibres.

(A) If the dimension of the fibres of $\pi$ is $r$ and $1 \leq r \leq 2$, then the dimension of the fibres of the semi-Riemannian submersion $\pi \circ \theta$ is less than or equal to 3 and greater than or equal to 2. By Theorem 1.1, $B$ is isometric to $\mathbb{H} H_{t}^{m}$ and $2 n+1=4 m+3,2 s+1=4 t+3$. Then $n=2 m+1, s=2 t+1$. By Theorem 3.14, we see that $\pi: \mathbb{C} H_{2 t+1}^{2 m+1} \rightarrow \mathbb{H} H_{t}^{m}$ is equivalent to the canonical semi-Riemannian submersion.

$(\mathrm{B})$ and $(\mathrm{C})$ If $B$ is an isotropic semi-Riemannian manifold or if index $(B) \in\{0, \operatorname{dim} B\}$, then, by Theorem $1.2, \pi \circ \theta$ is equivalent to one of the following canonical semi-Riemannian submersions:

$$
\begin{aligned}
& H_{2 t+1}^{2 m+1} \rightarrow \mathbb{C} H_{t}^{m}, \quad 0 \leq t \leq m \\
& H_{4 t+3}^{4 m+3} \rightarrow \mathbb{H} H_{t}^{m}, \quad 0 \leq t \leq m \\
& H_{7+8 t}^{15} \rightarrow H_{8 t}^{8}(-4), \quad t \in\{0,1\}
\end{aligned}
$$

If the dimension of the fibres of $\pi$ is greater than or equal to 3 , then the dimension of the fibres of $\pi \circ \theta$ is greater than or equal to 4 . Hence, in this case, $\pi \circ \theta$ is equivalent to $H_{7+8 t}^{15} \rightarrow H_{8 t}^{8}(-4), t \in\{0,1\}$. For $t=1$, the semi-Riemannian submersion $\pi$ is, after a change of signs of the metrics on the total space and on the base space, of type $\pi: \mathbb{C} P^{7} \rightarrow S^{8}(4)$. For $t=0, \pi$ is of type $\pi: \mathbb{C} H_{3}^{7} \rightarrow H^{8}(-4)$. In [Ran1] (for case $t=1$ ) and Ba-Ia (for case $\mathrm{t}=0$ ), it is proved that there are no such semi-Riemannian submersions 
with totally geodesic fibres. We proved that the dimension of fibres of $\pi$ is less than or equal to 2 .

Proof of Theorem 1.4. We suppose that there are such semi-Riemannian submersions. It is well-known that any quaternionic submanifold in $\mathbb{H} H_{s}^{n}$ is totally geodesic. Let $\eta$ : $H_{4 s+3}^{4 n+3} \rightarrow \mathbb{H} H_{s}^{n}, \xi: \mathbb{C} H_{2 s+1}^{2 n+1} \rightarrow \mathbb{H} H_{s}^{n}$, be the canonical semi-Riemannian submersions. By Theorem 2.5 in [Esc2, we see that $\pi \circ \eta: H_{4 s+3}^{4 n+3} \rightarrow B$ is a semi-Riemannian submersion with connected totally geodesic fibres. We remark that the dimension of the fibres of $\pi \circ \eta$ is greater than or equal to 4. Thus, by Theorem 1.2, we see that $\pi \circ \eta$ is equivalent to the canonical semi-Riemannian submersion

$$
H_{7}^{15} \rightarrow H^{8}(-4), \text { or } H_{15}^{15} \rightarrow H_{8}^{8}(-4)
$$

It follows that $\pi$ is one of the following types:

(i) $\pi: \mathbb{H} H_{1}^{3} \rightarrow H^{8}(-4)$, or

(ii) $\pi: \mathbb{H} H_{3}^{3} \rightarrow H_{8}^{8}(-4)$.

In Ucc, Ucci proved that there are no Riemannian submersions with fibres $\mathbb{H} P^{1}$ from $\mathbb{H} P^{3}$ onto $S^{8}(4)$. Therefore, Case (ii) is not possible.

The fibres of semi-Riemannian submersion $\pi \circ \xi: \mathbb{C} H_{3}^{7} \rightarrow H^{8}(-4)$ are totally geodesic by Theorem 2.5 in [Esc2], and complex submanifolds, since the horizontal lifting of the tangent space of the quaternionic line $\pi^{-1}(\pi(p))$ is invariant under the canonical complex structure on $\mathbb{C H}_{3}^{7}$. By [Ba-Ia], there are no semi-Riemannian submersions with complex totally geodesic fibres from $\mathbb{C} H_{3}^{7}$ onto $H^{8}(-4)$. Thus Case (i) is impossible.

\section{REFERENCES}

[Ba-Ia] G. Bădiţoiu and S. Ianuş, Semi-Riemannian submersions from real and complex pseudohyperbolic spaces, Differential Geom. Appl. 16(2002), 79-94.

[Ba-Ro] M. Barros and A. Romero, Indefinite Kähler manifolds, Math. Ann. 261(1982), 55-62.

[Bes] A. L. Besse, Einstein manifolds, Springer-Verlag, Berlin, 1987.

[Bis] R. L. Bishop, Clairaut submersions, Differential geometry (in honor of K. Yano), 21-31, Kinokuniya, Tokyo, 1972.

[Ehr] C. Ehresmann, Les connexions infinitésimales dans un espace fibré différentiable, Colloque de Topologie, Bruxelles 1950, p. 29-55, Georges Thone, Liège; Masson et Cie., Paris, 1951.

[Esc1] R. Escobales, Riemannian submersions with totally geodesic fibers, J. Differential Geom. 10(1975), 253-276.

[Esc2] R. Escobales, Riemannian submersions from complex projective spaces, J. Differential Geom. 13(1978), 93-107.

[Gra] A. Gray, Pseudo-Riemannian almost product manifolds and submersions, J. Math. Mech. 16(1967), 715-737.

[G-G1] D. Gromoll and K. Grove, The low-dimensional metric foliations of Euclidean spheres, J. Differential Geom. 28(1988), 143-156.

[G-G2] D. Gromoll and K. Grove, A generalization of Berger's rigidity theorem for positively curved manifolds, Ann. Sci. École Norm. Sup. (4)20(1987), 227-239. 
[Ian] S. Ianus, Differential geometry with applications to the theory of relativity, (in romanian) Ed. Academiei Române, Bucureşti, 1983.

[Mag] M. A. Magid, Submersions from anti-de Sitter space with totally geodesic fibres, J. Differential Geom. 16(1981), 323-331.

[One1] B. O'Neill, The fundamental equations of a submersion, Michigan Math. J. 13(1966), 459-469.

[One2] B. O'Neill, Semi-Riemannian geometry with applications to relativity, Academic Press, New York, London, 1983.

[Ran1] A. Ranjan, Riemannian submersions of spheres with totally geodesic fibres, Osaka J. Math. 22(1985), 243-260.

[Ran2] A. Ranjan, Riemannian submersions of compact simple Lie groups with connected totally geodesic fibres, Math. Z. 191(1986), 239-246.

[Rec] H. Reckziegel, A fibre bundle theorem, Manuscripta Math. 76(1992), 105-110.

[Ucc] J. Ucci, On the nonexistence of Riemannian submersions from $\mathbb{C} P(7)$ and $\mathbb{H} P(3)$, Proc. Amer. Math. Soc. 88(1983), 698-700.

[Wil] F. Wilhelm, The radius rigidity theorem for manifolds of positive curvature, J. Differential Geom. 44(1996), 634-665.

[Wilk] B. Wilking, Index parity of closed geodesics and rigidity of Hopf fibrations, Invent. Math. 144(2001), 281-295.

[Wol] J. Wolf, Spaces of constant curvature, McGraw-Hill Inc., New York, 1967.

Institute of Mathematics of the Romanian Academy

P.O. Box 1-764

BUCHAREST 014700

ROMANIA 